\title{
Solid-state NMR approaches for studying the interaction of peptides and proteins with membranes ${ }^{1}$
}

\author{
Anthony Watts * \\ Biomembrane Structure Unit, Biochemistry Department, Oxford University, Oxford OXI 3QU, UK
}

Received 18 March 1998; received in revised form 4 June 1998; accepted 5 June 1998

\section{Contents}

1. Introduction $\ldots \ldots \ldots \ldots \ldots \ldots \ldots \ldots \ldots \ldots \ldots \ldots \ldots \ldots \ldots \ldots$

2. Solid-state NMR for studying lipid-protein interactions in membranes $\ldots \ldots \ldots \ldots$

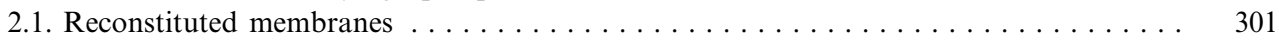

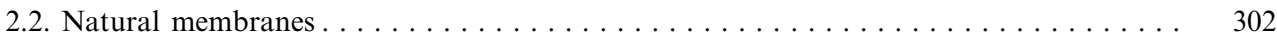

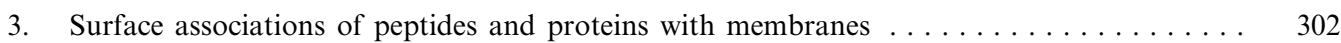

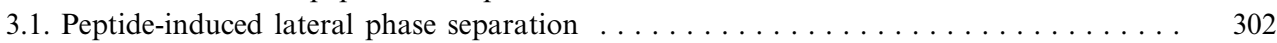

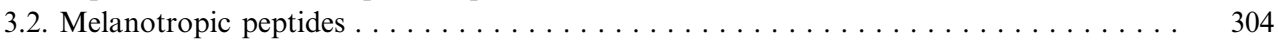

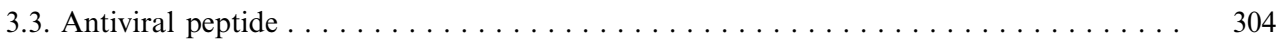

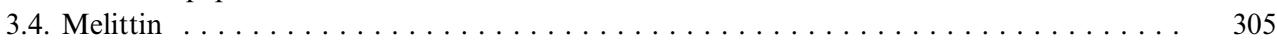

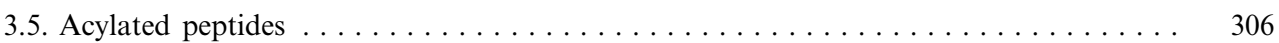

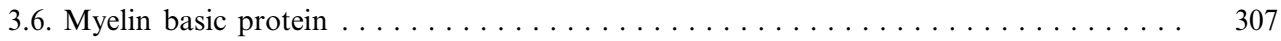

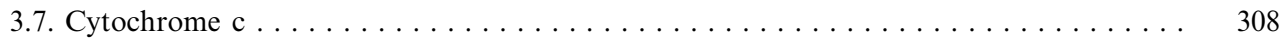

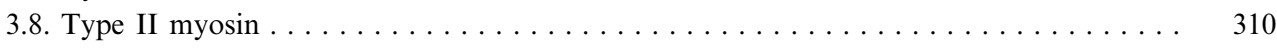

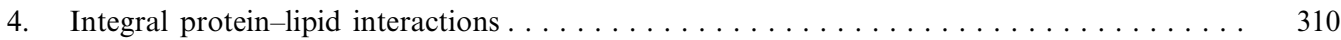

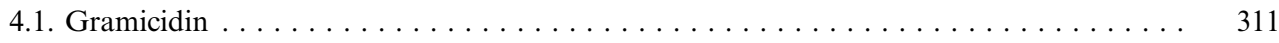

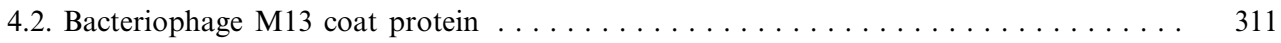

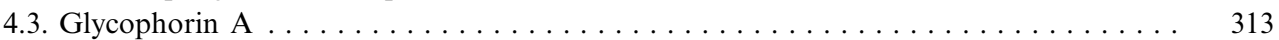

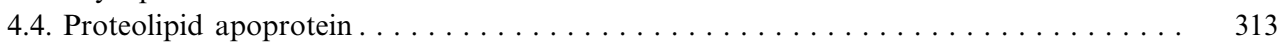

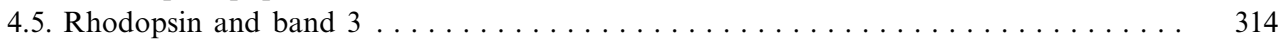

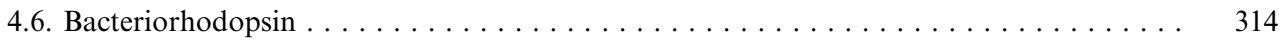

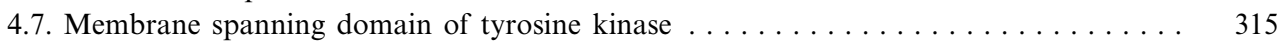

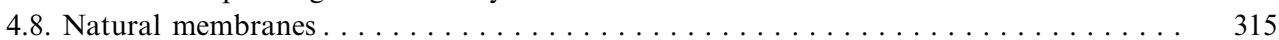

Abbreviations: NMR, nuclear magnetic resonance; MAS, magic angle spinning; DMPC, 1,2-dimyristoyl-sn-phosphocholine; DMPG, 1,2-dimyristoyl-sn-phosphoglycerol; PE, phosphatidylethanolamine; PG, phosphatidylglycerol; CL, cardiolipin

* Fax: +44-1865-275234; E-mail: awatts@bioch.ox.ac.uk; http://www.bioch.ox.ac.uk/ awatts/

1 Dedicated to Laurens van Deenen, who always took an interest in our work, and stimulated me to compile and edit the volume Protein Lipid Interactions which was published in 1993 as part of the New Comprehensive Biochemistry Series vol. 25, Elsevier, Amsterdam, for which he was a series editor. 


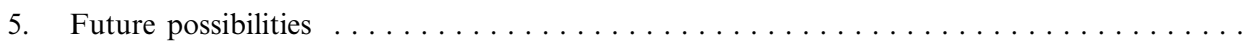

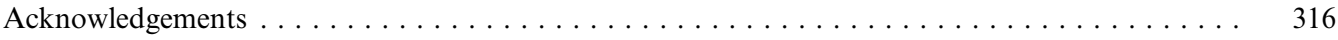

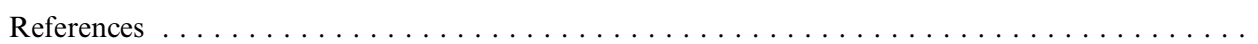

\section{Introduction}

The competent functioning of a biomembrane is controlled by both the protein and the lipid, mainly phospholipid, components [1-5]. The study of protein-lipid interactions in membranes is usually, therefore, the identification and description at the molecular level of a detectable interaction between one component and the other and, in the ideal case, a means of interpreting the structural information in such a way as to enable a description of the functional consequences of their mutual interactions.

In most biological membranes in viable cells, the composition and activity of the membrane is at least adequate, if not optimized through continual evolutionary constraints and influences, for the particular function being performed. To elucidate any intermolecular interactions between the various components, it is frequently necessary to exploit a probe placed in the system so that the nature, origin and type of molecular interactions involved can be identified, often spectroscopically [6-9] or genetically [10,11]. This is no more true than in magnetic resonance studies of protein-lipid interactions in membranes, where the spectroscopic property to be observed and exploited is used to report on the interactions, at the molecular level, and the probe may have to be introduced into the membrane or even more drastically, the membrane may have to be built around the reporter in reconstitution studies.

It is at the interface between a membrane protein and the bilayer that a transmembrane spanning protein is sealed into a membrane [12]. Most ion gradients which are set up by active transport protein, are subsequently used for driving secondary transport processes. If the ion gradients are then lost too quickly by non-specific leakage, which occurs anyway, through membrane regions at the proteinlipid interface, then energy (in the form of the potential energy due to the ion gradient) will be lost unnecessarily and not be converted into useful work. Molecular mis-match between the bilayer and the transport species does exist simply as a result of their different architecture and spatial character (lipid chains and protein residues), and so this interface could be one site of non-specific leakage.

Another consequence of protein-lipid interactions may be the mutual dynamic influence of one component on the other [13]. It is possible that a fluidity window is required within the bilayer part of a membrane for the proteins to undergo the requisite rates and degrees of molecular motion around the active site for biochemical activity to take place. Some proteins (type II, ion translocating ATPases, for example) undergo significant conformational changes during ion translocation; this gross molecular rearrangement may not be possible in a solid matrix. It is the lipid component of such bilayers that provide this fluidity window and changes in this component can thereby also alter the protein activities. Such interactions are likely to occur within the bilayer hydrophobic core whose chemistry cannot readily or quickly be altered in most situations, although head-group interactions with ions and charged species can modulate the bilayer chain motions rather dramatically and quickly $[14,15]$.

Any hydrophobic molecule which partitions into a biomembrane will diffuse rapidly throughout the bilayer and the site of action for a lipophile which can perturb or modulate the biological activity of a membrane, could well be the molecular interface between the protein and bilayer [16]. Such a region of mismatch could not only be a site for concentration of lipophiles, but also be the site of entry into a membrane spanning protein. Perturbation of the protein activity may be as a result of direct competition with lipid sensitive sites which themselves may be responsible for maintaining the $2^{\circ}, 3^{\circ}$ or $4^{\circ}$ structure of the protein in a form ready for activity.

Many spectroscopic studies of protein-lipid interactions are carried out to identify the structural aspects of the associations of lipids with proteins by exploiting the different motional properties of the lipids in the bulk bilayer and those at the protein- 


\section{NMR property}

\section{Membrane process}

\section{(LOG SECONDS)}

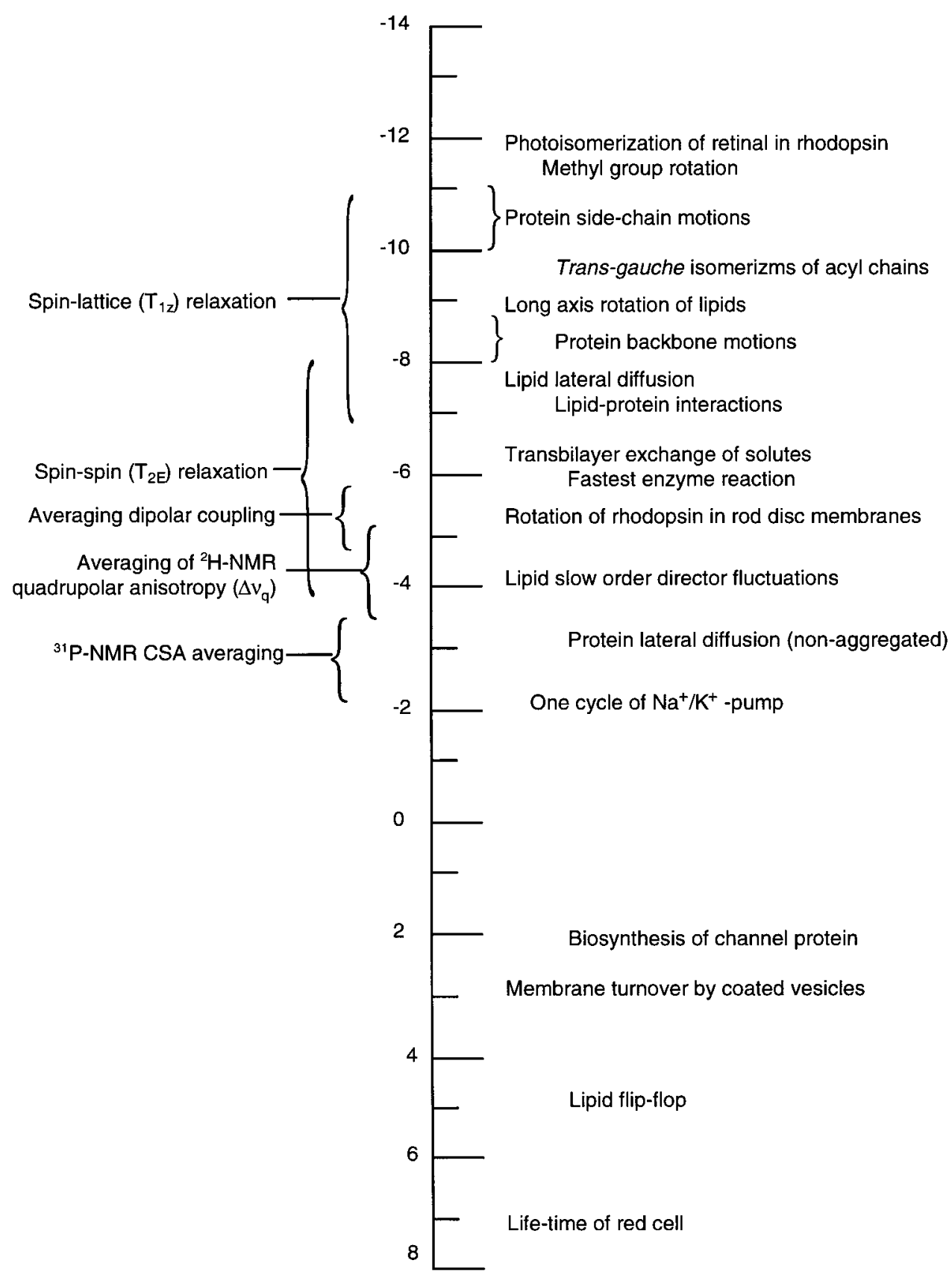

Fig. 1. A number of dynamic processes within biological membranes (right) can be accessed by judicious exploitation of an appropriate magnetic resonance property (left). The interaction of lipids with proteins in membranes which can be studied by such methods are those which alter the motional (either rate or amplitude) or conformational properties of the lipid chains or head groups and can thus be resolved by spectral changes which reflect those perturbations. At any one time, a number of motional changes over a range of dynamic time scales may be induced in a lipid upon interaction with a protein (and vice-versa), and thus the spectroscopic means for detecting and quantifying that interaction must be chosen rationally. In addition, the different kinds of mutual intermolecular perturbations of lipid and protein upon interaction may be transferred through the membrane by lipid or protein diffusion, and so one type of interaction may appear fast (averaged) on one time scale but slow (discrete) when viewed by another feature of a spectroscopic technique. Some properties are dependent upon the Lamour frequency. (Adapted from Ref. [21]; some data from Ref. [91].) 
bilayer interface which reveal themselves in the magnetic resonance spectrum. Too little effort has, unfortunately, been directed at correlating the structural and functional aspects of the information gained. This may often be as a result of the major difficulty in membrane work of controlling the biochemistry of the systems under study; preventing protein aggregation, resolving reconstitution technology where required, and ensuring the protein is active and correctly folded under the experimental conditions, which are all essential pre-requisites in such studies.

Here, the use of solid-state nuclear magnetic resonance as a tool to study lipid-protein interactions, will be reviewed and discussed, mainly in the light of recent information. Comprehensive reviews can be found elsewhere [17-30]. The approach does not have the same longer history in the area as other methods, such as fluorescence, DSC and spin-label ESR (see other papers in this issue). As a result, the number of systems studied using NMR is smaller, not least because of the less commonly available specialized instrumentation required, and the larger amount (mM) of sample required. No attempt is made to include a discussion of the wealth of information on membrane peptide and protein structure, and ligand to membrane receptors and transporters, from solid-state NMR [31-33] except where this gives new insights to lipid-protein interactions. Finally, the time scale of the more readily accessible NMR parameters (quadrupolar anisotropy averaging, chemical shift anisotropy) for membrane systems, is less appropriate to the faster molecular motions of lipid chains and protein residues when compared with spin-label ESR and fluorescence, something which can be an advantage or an obstacle to molecular interpretations of lipid-protein interactions.

\section{Solid-state NMR for studying lipid-protein interactions in membranes}

Lipid-protein specificity, exchange rates and stoichiometry are required for a complete description of lipid-protein interactions at the structural and molecular level [7,17-19,21,29,30,34,35]. NMR has an inherent time scale which is slower than many other spectroscopic methods [21] (Fig. 1), including optical methods and spin-label ESR [36]. Therefore, the type of information obtained will depend upon which parameter out of the NMR spectrum is exploited [3739]. For example, averaging deuterium quadrupolar interactions requires rates of molecular motions of $\sim 10-100 \mathrm{kHz}$, whereas for averaging of the phosphorus-31 chemical shift anisotropy, this rate is $\sim 4$ $6 \mathrm{kHz}$, depending upon spectrometer frequency. These rates are much slower than lipid diffusion, but in the range of protein diffusion [40]. Relaxation times are, however, more appropriate to lipid motion, with spin-lattice $\left(T_{1}\right)$ relaxation being in the ns- $\mu$ s range, and spin-spin $\left(T_{2}\right)$ relaxation in the ms range and more appropriate to slow bilayer director fluctuations.

Only a limited number of different NMR-active nuclei have been routinely exploited in solid-state NMR of lipid-protein interactions in biomembranes. These are deuterium, phosphorus-31 and, less often carbon-13. Fluorine-19 has been incorporated into proteins and lipid chains at specific sites [41-43]. Protons, despite their abundance and high sensitivity, have not proved to be a good nucleus because the strong dipolar coupling $(\sim 100 \mathrm{kHz})$ is very large and cannot readily be averaged by any means to give the high resolution information required for structural studies; this has just been solved in a new approach which has found application only in protein and lipid structure studies so far [44,45], but does have potential for studying lipid-protein interactions, especially for studying specific lipid moieties of a lipid or a protein residue involved in their mutual interactions. Nitrogen-15 is being exploited for studies of membrane peptides and proteins to good effect [46], but despite the occurrence of nitrogen in lipid head groups, the use of this nucleus for studying lipid-protein interactions has not yet been extensively demonstrated.

Being highly anisotropic in their molecular motion, membrane-embedded proteins and lipids containing any one of these observable NMR nuclei give rise to broad, spherically averaged powder patterns. Two approaches have been used to extract useful molecular information about a lipid (or protein) when interacting with a protein (or lipid) in a biomembrane. These two approaches are: static, solid-state NMR, where the anisotropy of the nucleus under study can be determined to give orienta- 
tional and dynamic information (Fig. 2); or magic angle spinning NMR (Fig. 3), where the anisotropic interactions can be averaged out by spinning the sample at the magic angle $\left(54.7^{\circ}\right)$ with respect to

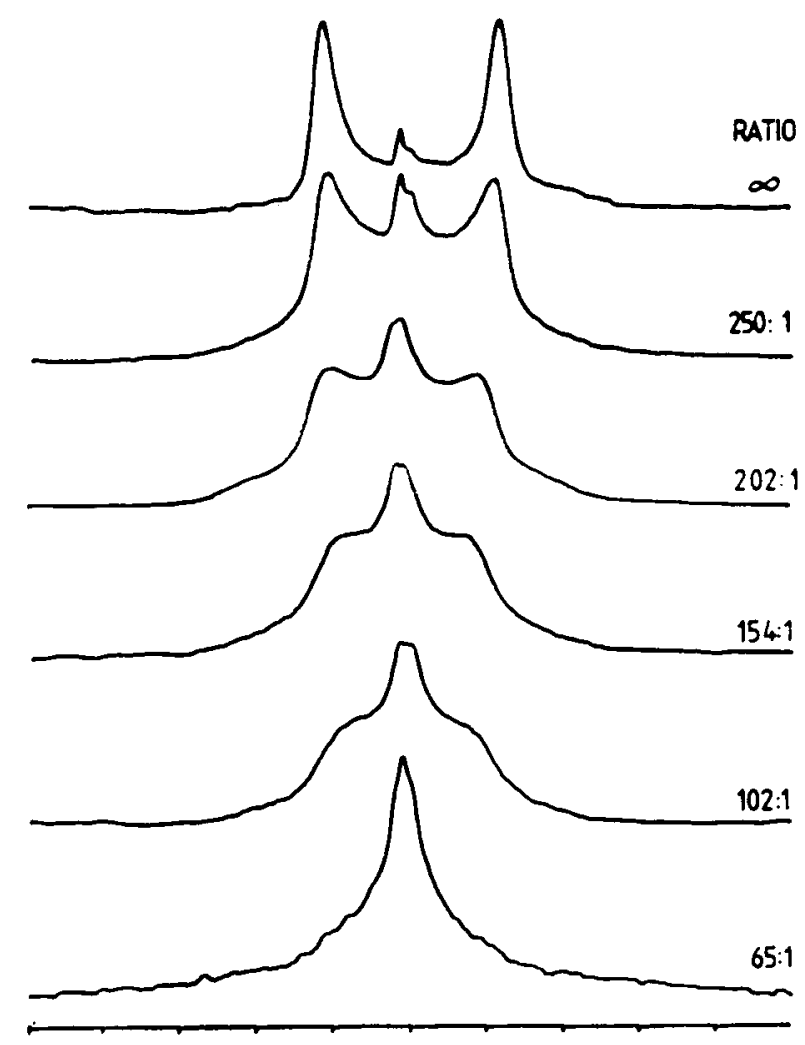

\section{$500 \mathrm{~Hz}$}

Fig. 2. An example of the deuterium-NMR spectral line-shapes and positional changes which occur when a single polypeptide chain, a monomeric integral membrane protein, rhodopsin $\left(M_{\mathrm{r}} \sim 39000\right)$, which rotates quickly $\left(\tau_{\mathrm{r}} \sim 20-100 \mu \mathrm{s}\right)$ in reconstituted liquid crystalline bilayers. Structural information about the labelled lipid segment is obtained from the line-positions for the spectral maxima (to give order parameters), and dynamic information is given by the relaxation times which are obtained from line-widths (to give spin-spin relaxation times, $T_{2}$ ) and spectral inversion recovery experiments (to give spin-lattice relaxation times, $\left.T_{1}\right)$. Here, the lipid (DMPC) is deuterated at the choline trimethyl group, $-\mathrm{N}^{+}\left(\mathrm{C}^{2} \mathrm{H}_{3}\right)_{3}$. Increasing the protein content collapses the well resolved spherically averaged spectrum for the pure, protein-free bilayers (upper spectrum) because the protein disorders the lipid head groups at the bilayer surface. The protein and lipid are in fast molecular exchange on the deuterium NMR anisotropy averaging time scale, therefore the perturbation of the bilayer by the protein is averaged throughout the whole bilayer. Rather less perturbation of the lipid acyl chains is observed when the deuterons probe the hydrophobic core. From Ref. [93].

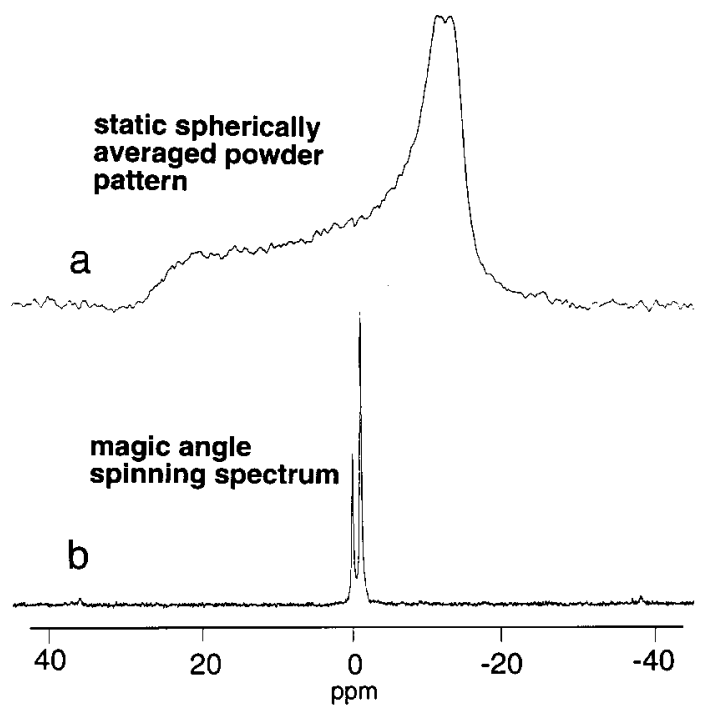

Fig. 3. Phospholipid bilayers give a broad, spherically averaged powder patterns in static, solid-state NMR methods (upper spectrum), and this line-shape is indicative of a bilayer; inversion and reduction in line-width indicates a hexagonal lipid phase, and an isotopic, single line indicates micelles, cubic or rhombic phases [47]. However, a new magic angle spinning approach [57] has now been shown to be useful in identifying specific phospholipid interactions with proteins, since each phospholipid component is resolved as a result of its chemical shift difference from other lipids, something which is not possible from the powder patterns which are $40-50 \mathrm{ppm}$ wide. Labelling is not now necessary and even natural membranes can be studied with this high-sensitivity nucleus.

the applied field, to give narrow spectral features from which chemical shift and (in certain cases) dipolar coupling, for use in structural, distance and dynamic studies.

Both approaches have been applied to the study of lipid-protein interactions, from which the molecular information needs to be obtained. This information is usually in the form of order parameters or relaxation times, giving structural and dynamic information, respectively. In addition, phase behaviour can be determined from the shape of the powder pattern with bilayers, micelles and hexagonal $\mathrm{H}_{\text {II }}$ phases being readily distinguished by phosphorus-31 NMR spectral shapes [47].

\subsection{Reconstituted membranes}

Reconstituted membranes are usually formed by detergent dialysis of a co-solubilized phospholipid- 
protein solution, such that protein insertion occurs into the bilayer $[48,49]$. Single or a small number of lipid components are routinely used and phosphatidylcholines being the most commonly used lipid for such work, although charged lipids may facilitate insertion of some proteins. In addition, protein can be added back to pre-formed bilayers, especially if these proteins interact at the membrane surface, often with anionic lipids.

In both of these cases, all the membrane lipid constituents can contain, for example, deuterons at any specific position in the lipid (head groups, glycerol backbone, or acyl chains) for NMR studies, or the indigenous phosphorus-31 nucleus can be exploited without the need for labelling. Although the broad powder patterns obtained for both of these methods contain little information about the specificity of lipid-protein interactions, new magic angle spinning (MAS) methods (Fig. 3) have recently been shown to yield specific molecular interactions with peripheral proteins (see below).

\subsection{Natural membranes}

Phosphorus-31 has not been extensively exploited in the study of lipid-protein interactions in natural membranes, other than for peripheral protein binding. The lack of specificity in assignment of individual phospholipids has only just been accomplished, in novel magic angle spinning NMR approaches (see below), thereby opening the way for identifying specific protein-lipid interactions for a particular lipid type with a protein of interest. Growth of bacteria on deuterated fatty acids has been used for the study by deuterium NMR (see below) in which the spectral parameters such as quadrupole splitting and linewidths, give structural and dynamic information in an averaged way, respectively. However, in general, the study of natural membranes, as with any approach, is complex as a result of the heterogeneous composition of the membranes, and difficulty in defining precisely which proteins may be interacting with which lipids, except in certain cases. The difficulty of incorporating nitrogen-15 into natural membranes, then identifying the individual components has hampered the use of this nucleus, although methods are now becoming available for labelling using expression methods.

\section{Surface associations of peptides and proteins with membranes}

Peptides and proteins associate with biomembrane surfaces in vivo, either prior to insertion and then translocation, or as a pre-requisite to assembly then insertion as a folded entity [50]. Protein pre-sequences, toxins, melittin and several synthetic peptides have been studied using NMR to elucidate such interactions, and any subsequent hydrophobic associations with the bilayer, and sometimes macroscopic alterations in the membrane structure [47].

Structural details at atomic resolution of how peripheral membrane proteins interaction with membrane surfaces are rare, and only recently have crystal structures been resolved for a range of lipases showing the binding sites of lipids which are substrates [51]. In addition, proteins which associate both hydrophobically and at the surface of biomembranes are being structurally resolved using isotopically labelled $\left({ }^{15} \mathrm{~N}\right)$ proteins and solid-state NMR methods [46]. In none of these studies have any details of specific lipid-protein interactions yet been described.

\subsection{Peptide-induced lateral phase separation}

One consequence of peptide-lipid associations is lateral domain formation [52], with peptide-lipid complexes segregated from lipid-rich areas. Such compartmentalization of domains within membranes may be important for functional reasons, giving local areas within some kinds of reaction can occur, and preventing others [52,53]. Solid-state NMR approaches have been useful in defining the life-time limits for laterally segregated areas, and are now showing promise in defining the size and composition in such areas.

\subsubsection{Polymyxin}

Polymyxin was shown to induce lateral phase separation of charged phospholipids from zwitterionic phospholipid in bilayers (phosphatidylglycerol from phosphatidylcholine), using deuterium NMR as a non-perturbing probe of the membrane surface [54]. No estimates of domain size were made, but the long life-time ( $>1 \mathrm{~ms}$ ) for the domains was demonstrated by the well resolved spectral differences between lip- 


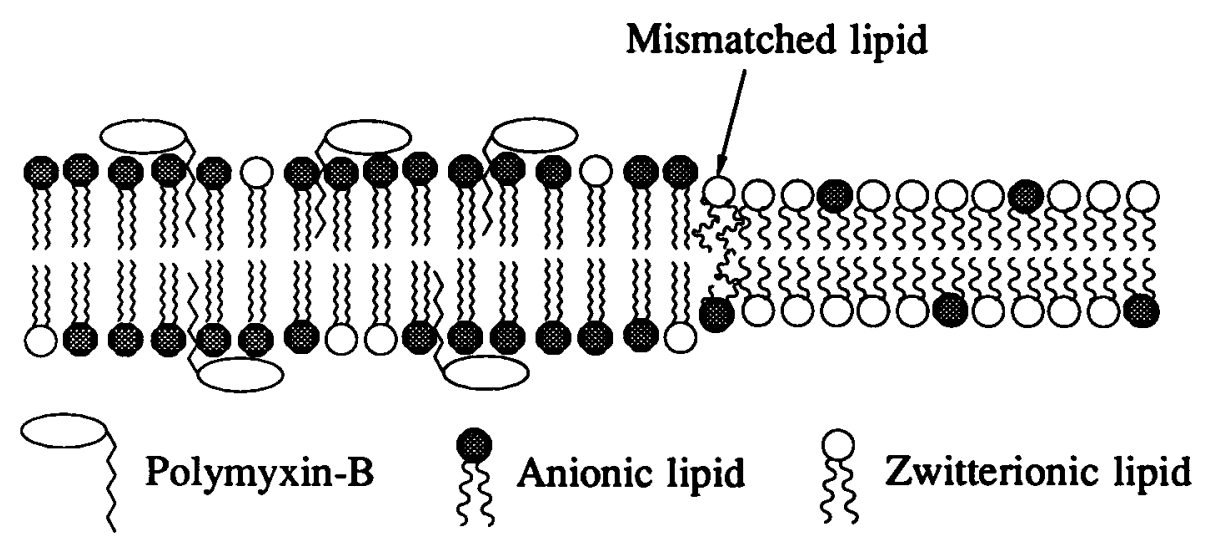

Fig. 4. A schematic representation of lateral phase separation for anionic lipids from zwitterionic lipids in a mixed lipid bilayer induced by the peptide antibiotic, polymyxin. Deuterium-NMR results imply a well-defined separation between two different phases, and the mis-match that occurs between the domains was interpreted as a local area of weakness which could permit bacterial cell lysis [54].

ids in a complex with the peptide, and those remote from the complex. This observation suggests a mode of action for such peptide antibiotics when associating with bacterial membranes rich in charged lipids, in inducing areas of weakness and mis-match in the plasma membrane of the bacterium (Fig. 4), thereby causing lysis and cell death.

\subsubsection{Cardiotoxin II}

A number of proteins and peptides have now been found to induce lateral phase separation of, usually charged, lipids in mixed lipid bilayers [52]. The interaction of the cationic protein cardiotoxin II with mixtures of zwitterionic and anionic (1:1) phospholipids in bilayers has been investigated using phosphorus-31 and deuterium (NMR) [55]. Adding cardiotoxin to the mixtures produced a two-component phosphorus-31 NMR spectrum, in which the second component had a decreased chemical shift anisotropy. Simultaneously, the deuterium NMR quadrupolar splitting measured from the head groups of the zwitterionic lipid head groups were reduced. It was concluded that cardiotoxin produces an altered macroscopic phase state of the lipid bilayers, and this obscures any effects on bilayer surface electrostatics observed by deuterium NMR, which was also observed in the melittin studies [56].

Applying MAS phosphorus-31 NMR spectroscopy to the bilayers [57], two isotropic resonances were resolved in the absence of cardiotoxin and were assigned to the two individual phospholipids [55]. Add- ing cardiotoxin produced two new isotropic resonances. Quantifying the intensities of the various resonance lines revealed that the binding isotherms for different phosphatidylcholine/phosphatidylglycerol mixtures follow classical binding characteristics when expressed as a function of the cardiotoxin/ charged lipid ratio. The results indicate that cardiotoxin binds preferentially to the anionic lipid and is able to segregate laterally the anionic and zwitterionic lipids from the remaining zwitterionic lipid. Fitting of the binding isotherms was achieved using a two-site model derived from statistical-thermodynamic considerations. One class of binding site is specific for phosphatidylglycerol and the other is non-specific, capable of binding both phosphatidylcholine and phosphatidylglycerol, which confirmed in the more recent work on pentalysine induction of lateral phase separation (see below) [58].

\subsubsection{Pentalysine}

To understand the electrostatic contribution of association of the MARKS protein (myristoylated alanine-rich $\mathrm{C}$ kinase substrate), the interaction of pentalysine, which is contained within the effector region of the protein, has been studied in its interaction with charged phospholipid bilayers [52]. To gain more insight into this interaction, high-resolution, MAS phosphorus-31 NMR of the phospholipids has been used to demonstrate a specific interaction of the charged lipids with the peptide, to form domains within the bilayers of a mixture of charge and un- 

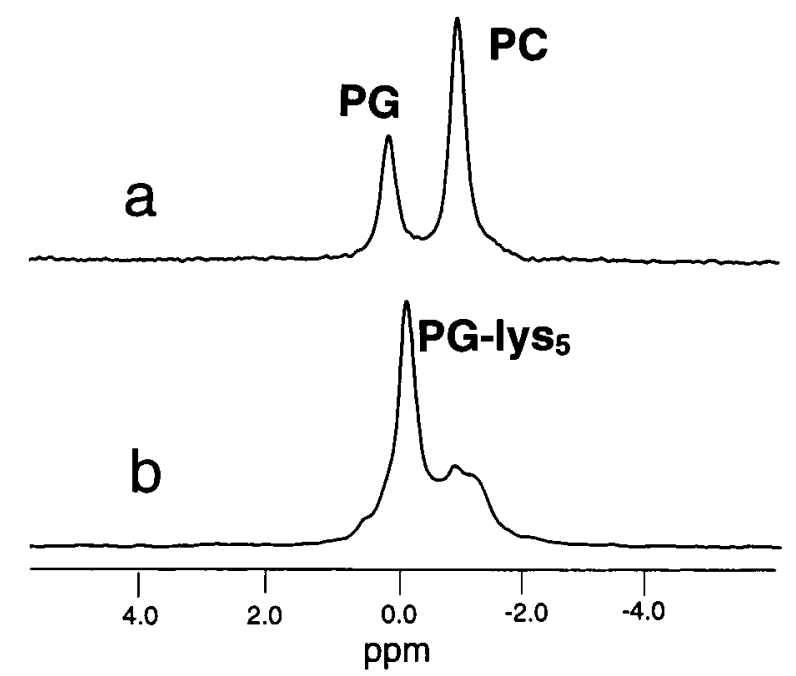

Fig. 5. Expanded phosphorus-31 MAS NMR spectra at high spinning speed $(6 \mathrm{kHz})$ at $298 \mathrm{~K}$ of phosphatidylglycerol/phosphatidylcholine (1:2 molar ratio) mixtures in the absence (a) and presence (b) of pentalysine, at concentrations of peptide (26:1 lipid/peptide molar ratio) at which lateral phase separation is induced. The narrow spectral lines permit some indication about which lipid is interacting with the peptide, in this mixed phospholipid bilayer system which in conventional static, phosphorus-31 NMR would only give a single, broad (40-50 ppm) spectral envelope which masks the chemical shift difference $(\sim 2 \mathrm{ppm})$ between the individual phospholipids (see also Fig. 3 and Ref. [57]. The new spectral component in b is from the PG-peptide complex, and the reduction in the PC resonance indicates that the peptide-lipid domain also contains PC and is not, therefore, solely composed of the anionic lipid alone. Domain sizes are estimated to be $>1-0.1 \mu \mathrm{m}$ and their life-time $>100 \mathrm{~ms}$ [58].

charged lipids [58] (Fig. 5). The regions of the bilayers free of the peptide association, were found to be free of charged lipids. From an analysis of the exchange rates of lipids into and out of the domains, and assuming the lateral diffusion rate of the lipids are in the $10^{-7}-10^{-8} \mathrm{~cm}^{2} \mathrm{~s}^{-1}$ range, gives an estimate for the upper limit of the domains to be $0.1-1 \mu \mathrm{m}$.

\subsection{Melanotropic peptides}

The interaction of the cationic tridecapeptide alpha-melanocyte stimulating hormone $(\alpha-\mathrm{MSH})$ and the biologically more active analogue (Nle-4,DPhe7)- $\alpha-\mathrm{MSH}$ with anionic and zwitterionic lipid membranes was investigated by deuterium NMR of headgroup labelled phosphatidylglycerol and spin-label ESR [59]. Both peptides decrease the motional free- dom of the acyl chains. No peptide effect was detected with neutral lipid bilayers. Changes in the quadrupole splittings and spin lattice relaxation time of phosphatidylglycerol deuterated at the glycerol head group paralleled the results obtained with ESR, showing that the peptides cause a better packing both at the head group and at the acyl chain bilayer regions. The greater change in membrane structure caused by the more potent analogue, when compared to the native hormone, was interpreted in terms of its stronger lipid association constant and/or its deeper penetration into the bilayer, thereby relating structural and functional consequences in this study.

\subsection{Antiviral peptide}

The influence of the antiviral peptide, carbobenzoxy-D-phenylalanyl-L-phenylalanylglycine (ZfFG), on the average conformation of phosphatidylcholine in bilayers was investigated with multinuclear solidstate NMR [60]. Phosphatidylcholine was specifically deuterated (separately) in the choline head group, the C-2 carbon of the acyl chains and at all the carbons of the acyl chains. Phosphatidylcholine was also synthesized with the carbonyl carbons of the ester bonds between the glycerol and the hydrocarbon chains enriched in ${ }^{13} \mathrm{C}$. Deuterium NMR of the phosphatidylcholine perdeuterated in the acyl chains showed a loss of intensity from the deuterium nuclei with the largest quadrupole splitting in the presence of $\mathrm{ZfFG}$, while the remainder of the powder pattern was largely unaffected. The phosphatidylcholine specifically deuterated at the C-2 carbon (representative of the $\mathrm{C}-\mathrm{D}$ bonds giving rise to the largest quadrupole splittings) showed the same loss of intensity, suggesting changes in the phospholipid conformational dynamics near the glycerol. Analysis of the powder patterns in the ${ }^{13} \mathrm{C}$-NMR spectrum of phosphatidylcholine labelled with ${ }^{13} \mathrm{C}$ in the carbonyl carbons revealed a significant change in the average orientation of the $s n-1$ carbonyl due to the presence of the ZfFG and no change in the $s n-2$ carbonyl orientation. Changes in the head-group conformation, as detected by deuterium NMR of the deuterons in the $\alpha$ and $\beta$-methylenes of the choline head group and phosphorus-31 NMR of the phosphate segment, reflected the electrostatic nature of the interaction of 
the carboxyl of ZfFG with phosphatidylcholine bilayers. No significant effect was observed from the deuterium nuclei in the $N$-methyls of the choline. From these data it was concluded that phosphatidylcholine had access to more than one conformation around the glycerol segment of the molecule in a bilayer. In the absence of ZfFG, the two carbonyls are inequivalent in their orientation. The antiviral peptide ZfFG favoured a conformation in which the average orientations of the two ester carbonyls (with respect to the axis of rotation diffusion) were approximately equivalent. This altered phospholipid conformation may be the source of the differences in phospholipid packing observed in the presence of the antiviral compound. The loss of apparent deuterium NMR intensity was likely due to the dynamics (on the time scale of $10^{-5} \mathrm{~s}$ ) of the interchange between the (at least) two conformations adopted by the phosphatidylcholine in the presence of ZfFG.

\subsection{Melittin}

The interaction of the amphiphilic toxin from bee venom, melittin, with dimyristoylphosphatidylcholine selectively deuterated in the choline head group has been studied by deuterium and phosphorus-31 NMR [56]. The action of residual phospholipase $\mathrm{A}_{2}$ in melittin samples resulted in mixtures of dimyristoylphosphatidylcholine and its hydrolytic products that underwent reversible transitions at temperatures between $30^{\circ} \mathrm{C}$ and $35^{\circ} \mathrm{C}$ from extended bilayers to micellar particles which gave narrow singe-line deuterium and phosphorus-31 NMR spectra. Similar transitions were observed in dimyristoylphosphatidylcholine and myristoyllysophosphatidylcholine (lysoPC)/myristic acid mixtures containing melittin but not in melittin-free mixtures, indicating that melittin is able to stabilize extended bilayers containing dimyristoylphosphatidylcholine and its hydrolytic products in the liquid-crystalline phase. Melittin, free of phospholipase $\mathrm{A}_{2}$ activity, and at 3-5 $\mathrm{mol} \%$ relative to DMPC, induced reversible transitions between extended bilayers and micellar particles on passing through the liquid-crystalline to gel phase transition temperature of the lipid, effects similar to those observed in melittin-acyl chain deuterated dipalmitoylphosphatidylcholine mixtures [61]. LysoPC at concentrations of $20 \mathrm{~mol} \%$ or greater relative to dimyristoylphosphatidylcholine induced transitions between extended bilayers and micellar particles with characteristics similar to those induced by me-

$(25 \circ \mathrm{C})$
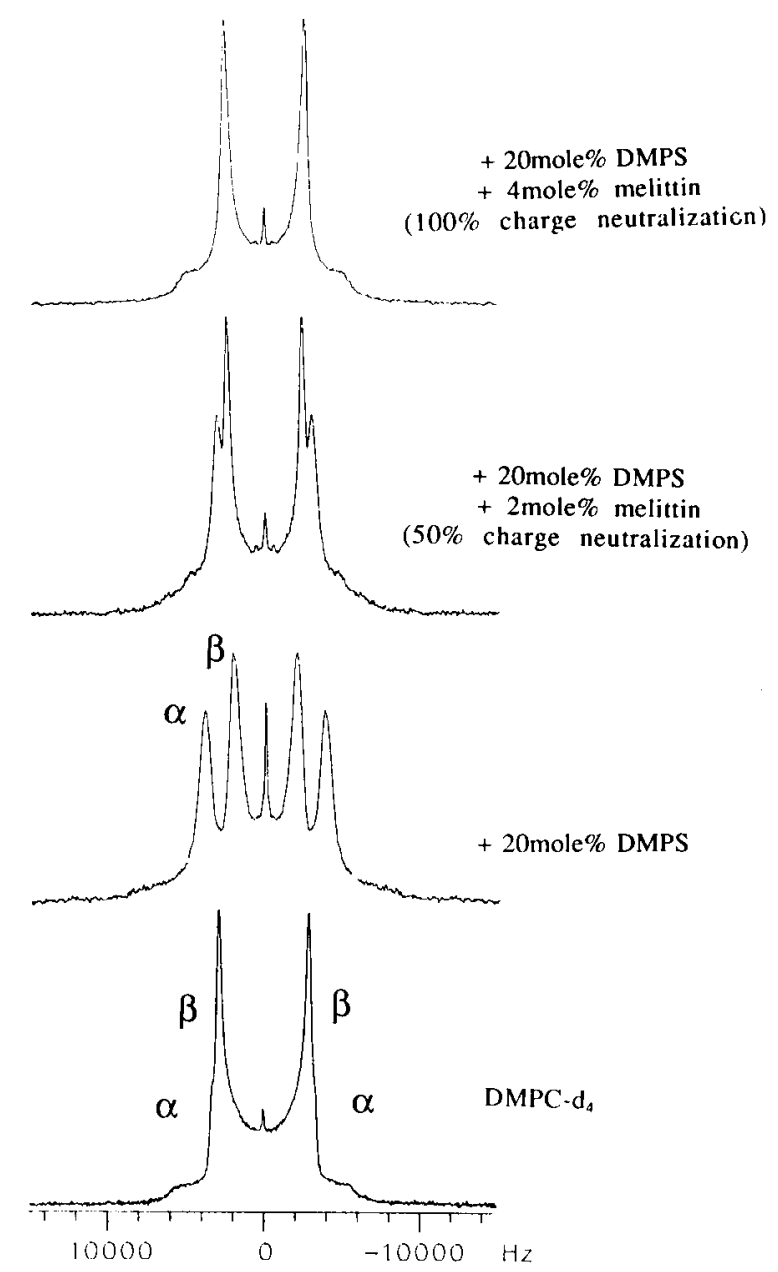

Fig. 6. The deuterium-NMR spectra for DMPC, deuterated at the two methylenes in the choline head group. These two labelled positions accidentally give rise to similar quadrupole splittings (spectrum a), but on the addition of (unlabelled) DMPS, the well established perturbation of the PC head group by DMPS $[64,65]$ is clearly revealed by the now clear resolution of the quadrupole splittings for the two labelled methylenes (b). On addition of melittin, to neutralize the DMPS head-group charge by $50 \%$ (spectrum c) and $100 \%$ (spectrum d), the deuterium-NMR spectrum from DMPC returns now to a very similar form seen in the absence of DMPS (spectrum a). This shows that the DMPC is predominantly excluded from the DMPS-melittin complex, as seen with other peptides (for example, pentalysine, see above [58] and polymyxin, see above [54]) by NMR. 
littin. It is proposed that these melittin- and lysoPCinduced transitions share similar mechanisms. The effects of melittin on the quadrupole splittings and $T_{1}$ relaxation times of head group-deuterated dimyristoylphosphatidylcholine in the liquid-crystalline phase, share features similar to the effects of metal ions on phosphatidylcholine head groups [62], indicating that the conformational properties of the choline head group in phosphatidylcholine bilayers may be affected by melittin and by metal ions in a similar manner.

The interaction of bee venom melittin with mixed phospholipid bilayers composed of dimyristoylphosphatidylcholine and dimyristoylphosphatidylserine deuterated in their head groups, separately, was studied in ternary mixtures by using deuterium NMR spectroscopy [63]. The changes in the deuterium quadrupole splittings of the head-group deuteromethylenes of phosphatidylcholine induced by dimyristoylphosphatidylserine in binary mixtures $[64,65]$ were systematically reversed by increasing concentrations of melittin, so that at a melittin concentration of $4 \mathrm{~mol} \%$ relative to total lipid the deuterium NMR spectrum from dimyristoyl phosphatidylcholine in the ternary mixture was similar to the spectrum from pure dimyristoylphosphatidylcholine bilayers (Fig. 6). This concentration of melittin is sufficient to neutralize the excess negative charge from dimyristoylphosphatidylserine. The absence of deuterium NMR signals arising from melittin-bound labelled dimyristoylphosphatidylserine in ternary mixtures indicates that the reversal by melittin of the effects of dimyristoylphosphatidylserine on the quadrupole splittings of dimyristoylphosphatidylcholine, results from the response of the choline head group to the net surface charge rather than from phase separation of melittin-anionic lipid complexes. In mixtures of the labelled zwitterionic and anionic lipids, melittin caused systematic changes in the quadrupole splittings of the dimyristoylphosphatidylserine head-group deuterons that closely matched effects observed for a cationic transbilayer polyleucyl peptide (K-2GL-20K-2A) in similar ternary mixtures [66]. The similarity in the effects of the two cationic but otherwise dissimilar peptides indicates that the dimyristoylphosphatidylserine head group responds to the surface charge resulting from the presence in the bilayer of charged amphiphiles, in a manner anal- ogous to the response of the choline head group of phosphatidylcholine to the bilayer surface charge. The presence of phosphatidylserine greatly stabilized phosphatidylcholine bilayers with respect to melittininduced micellization, indicating that the latter effect of melittin may not be important for the haemolytic activity of the peptide.

\subsection{Acylated peptides}

The association of two peptides which are myristoylated to the N-terminal glycine residue and with sequences that are conserved among many myristoylated proteins, has been studied by deuterium NMR. Both peptides contain the obligatory glycine residue at the N-terminal for acylation. One peptide (SerPep) contained a serine at the 5 th position to resemble the sequence of known, membrane-associating proteins such as the $\alpha$-transducin subunit, VP4 from polio virus, and $\mathrm{p} 60^{\mathrm{src}}$. The other peptide (ThrPep), had threonine at the 5th residue to resemble a leukaemia virus $\mathrm{p} 15^{\mathrm{gag}}$ protein and polyprotein Pr65 $5^{\mathrm{gag}}$. In solution, the peptides did not shown any association, which is in keeping with other studies of membrane

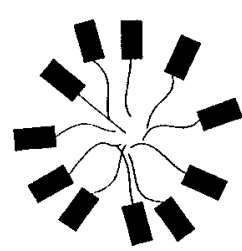

c

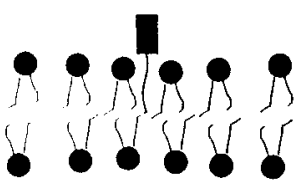

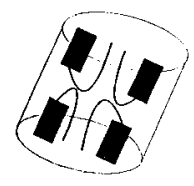

d

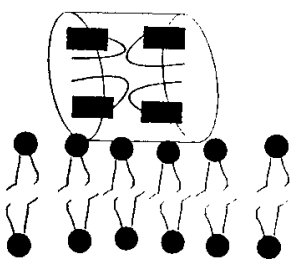

Fig. 7. There are as significant number of acylated proteins, both integral and 'peripheral', whose interaction with the membrane is not fully understood. In a model study, the mode of membrane interaction for two peptides has been studied using acylated SerPep (a) and ThrPep (b), when in solution (a,b) and when interacting with membranes (c,d). The acylated SerPep, by virtue of the association of the amino acid residues and the phospholipid head groups, does not have the acyl-peptide chains of a complex intercalated into the bilayer, in contrast to the acylated SerPep whose chains do penetrate the bilayer. The stoichiometry of the complexes are not known, and the diagram is illustrative only [66]. 
peptides studied by solution state NMR. In contrast, deuterium NMR spectra show that the acylated (perdeuterated, $\mathrm{d}_{27}$ ) SerPep penetrates into the hydrophobic core of the bilayer, whereas the other peptide, ThrPep, binds through protein residue interactions with the lipid head groups, with the acyl chains in this case, stabilizing a peptide aggregate at the bilayer surface by forming a hydrophobic core [67] (Fig. 7).

To gain insight into the structural consequences of acylation for membrane proteins, palmitic acid has been covalently attached to the ethanolamine end of gramicidin A (gA), which functions as a well-characterized cation-selective membrane channel [68]. Deuterium NMR spectra for labelled samples in hydrated dimyristoylphosphatidylcholine (DMPC) bilayers show that, for the major gA conformation, the deuterated side chains of Trp9 and Leu10 are markedly influenced by acylation, whereas the side chains of Val7 and Trp11 are essentially unaffected. The NMR results in both environments suggest that the indole ring of Trp9 is situated near the side chain of Leu10 and moves away upon acylation. It is therefore proposed that acylation provides a subtle mechanism to modulate protein and lipid interactions and to regulate the stability and function of proteins within membranes.

\subsection{Myelin basic protein}

The specificity of lipid-protein interactions with peripheral proteins has been demonstrated by solidstate NMR for a small number of examples, the ones being studied are usually chosen because of their availability. Myelin basic protein from bovine spinal cord was shown to have a specific interaction with charged phospholipids in bilayers of charged and zwitterionic lipids, from deuterium NMR studies in which the lipid head groups were labelled [69]. The mode of association was shown to be rather specific in its stoichiometry, with one protein residue associating with one lipid.

Also, it was shown that lipid exchange with the myelin basic protein-lipid complex was fast ( $v_{\mathrm{ex}}<\mathrm{kHz}$; whereas $v_{\mathrm{ex}} \sim 100 \mathrm{MHz}$ for lipid-lipid exchange) on the deuterium NMR time scale, and confirmed the dynamic nature of lipid protein interaction. Fast exchange between two the distinct sites was described simply by $X($ observed $)=A$. $X$ (at A) $+B . X$ (at B), where $A$ and $B$ are the proportions of the reporting species in each of the two sites $A$ and $\mathrm{B}$, and $X$ is some relaxation rate or spectral frequency of the system for NMR; one site (A) is usually 'free,' and the other (B) is a 'boundary' site. Small-molecule binding to proteins by proton NMR, fluorescent quenching of intrinsic chromophoric groups in proteins by labelled lipids in membranes, has also been modelled in a similar way $[70,71]$ and lipid-protein exchange, as determined by deuterium NMR $[63,68,69,72,73]$. One complication to any similar 'binding' studies, is that analysis is only appropriate over a range where linearity exists (Fig. 8), and if any protein aggregation, or the nature of the binding changes during the titration, then a simple formulation does not hold.

For the myelin basic protein study, the spectral parameter chosen was the quadrupole splittings

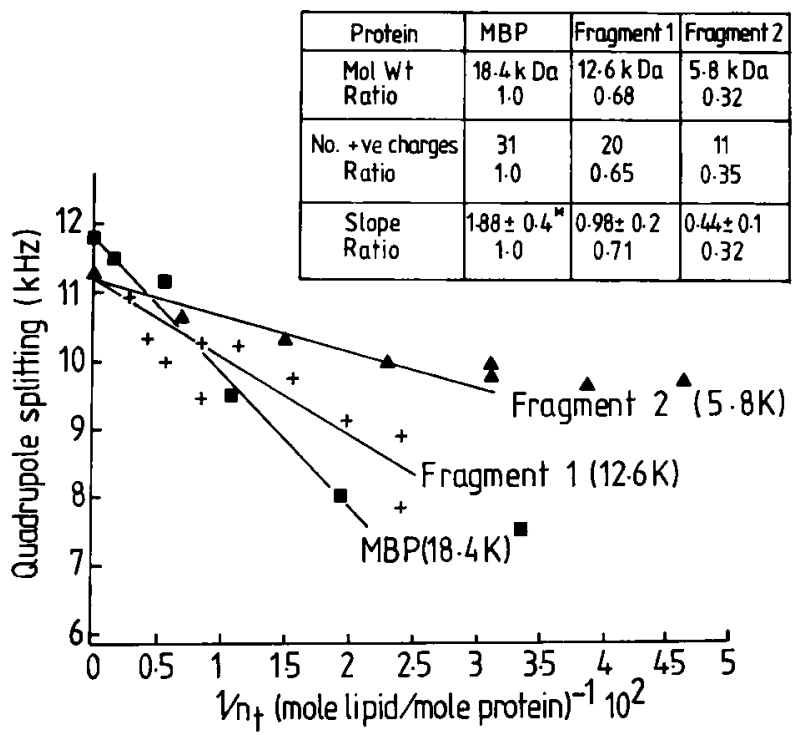

Fig. 8. Deuterium solid-state NMR studies of head group (glycerol-d $\mathrm{d}_{5}$ )-labelled phosphatidylglycerol have been undertaken to demonstrate that the binding of myelin basic protein $\left(M_{\mathrm{r}} \sim 18400\right)$, and its two major fragments $\left(M_{\mathrm{r}} \sim 12600\right.$; $\left.M_{\mathrm{r}} \sim 5800\right)$, produced by proteolytic cleavage to anionic bilayers, produces complexes in which the protein-lipid exchange is fast on the deuterium NMR time scale. The linearity of the measured quadrupole splittings with protein/lipid ratio, shows that protein aggregation and lateral phase separation does not occur, on this time scale, and the slopes, which are related to the charge density on each peptide, show that the charge distribution along the peptide fragments is similar to that for the intact protein [74]. 
from the head-group deuterons in each of the two lipid types. No long-lived (with a life-time of longer than $0.1 \mathrm{~ms}$ ) domains were therefore observed, and the nature of the association was later shown to be similar for the two major fragments of the protein (Fig. 8), confirming a reasonably uniform distribution of charges along the protein and its fragments [74].

\subsection{Cytochrome c}

The complex conformational transitions induced by cytochrome $c$ upon interaction with phospholipid bilayers has been demonstrated using a range of various solid-state NMR methods. Here, the well-established crystal structure of the protein in solution, clearly does not exist when the protein is interacting with the membrane surface, and shows the power and need to carry out spectroscopic studies on such interactions, even though the crystal structure is known. Deuterium NMR of head-group labelled cardiolipin revealed the induction of slow order director fluctuations of the membrane as a result of cytochrome $c$ binding [75]; these fluctuations result in bilayer undulations with a frequency in the $10^{-3}-1$

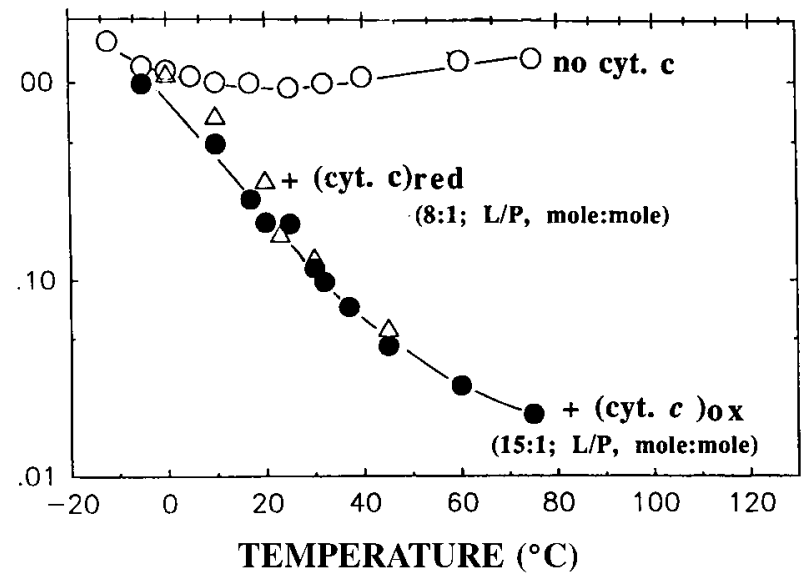

Fig. 9. The variation (on a log scale) in spin-lattice relaxation time (ordinate) with temperature for bilayers of cardiolipin alone (open circles) and when complexed with ferricytochrome $c$ (closed circles) and ferrocytochrome $c$ (open triangles) at lip$\mathrm{id} /$ protein molar ratios of $8: 1$ and $26: 1$, respectively. The characteristic minimum in $T_{1}$ for pure lipid bilayers is reduced and significantly (by $\sim 100$ ) enhanced when protein is present, indicating that the change is not due to motional changes, but due to a direct paramagnetic interaction of the heme of the protein with the phosphate [77].
13C CP-MASS SPECTRA FOR HYDRATED CARDIOLIPIN BILAYERS $\left(25^{\circ} \mathrm{C}\right)$

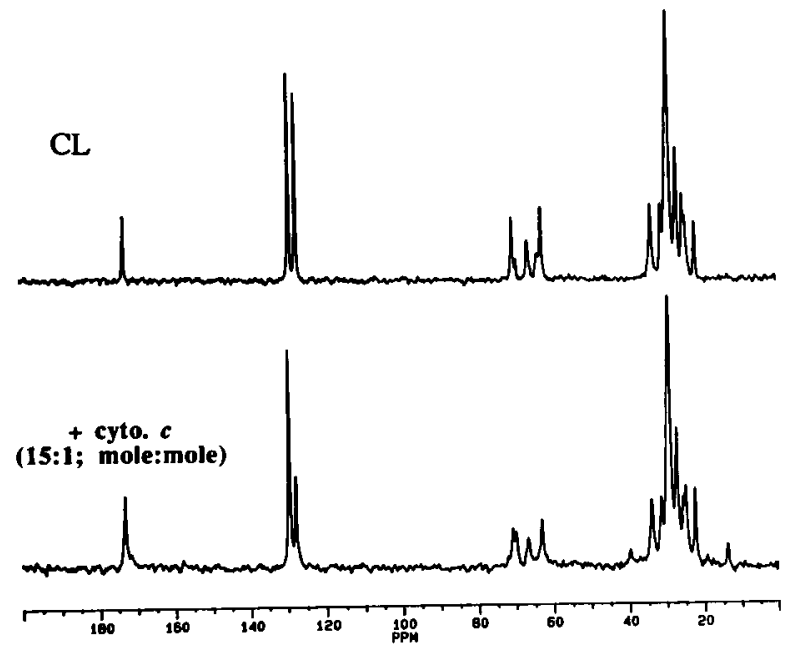

Fig. 10. Natural abundance, proton decoupled cross-polarization MAS ${ }^{13} \mathrm{C}-\mathrm{NMR}$ spectra of hydrated liquid crystalline, bovine heart cardiolipin bilayers without (a) and with (b) bound cytochrome $c$, at a 1:15 protein/lipid molar ratio. In the presence of the protein, the intensity of certain of the lipid resonances are reduced, indicating some selective interaction of the protein with the linoleoyl ( $c i s-18: 2-\Delta^{9,13}$ ) groups of the fatty acyl chains, suggesting that the protein induces a greater degree of large amplitude motions within this part of the bilayer, even though it is primarily peripherally bound [78].

s range, in keeping with other similar observations (see below; Section 4.8).

Even though the heme moiety is liganded through met-80, and a stable form in the crystal structure, phosphorus-31 NMR relaxation measurements for the cardiolipin lipid phosphates showed that the heme group makes contact with the phosphates in a dynamic and fast (rate is $<\mathrm{ms}$ ) way [76,77] (Fig. 9 ), and that some interaction does occur with the lipid chains, even though the protein is peripherally bound [78] (Fig. 10). This observation would imply a partial unfolding of the protein which was confirmed by solid-state NMR studies of the protein which was labelled at specific places (lysines, histidine and ethanolamine groups, separately). For example, rotational diffusion on the membrane surface was not fast enough to account for mobility in the protein residues carrying labels (which is most of the protein) and could only be explained by considerable config- 
urational distortions induced upon protein binding. Indeed, a strong binding of protein with liquid-crystalline bilayers of cardiolipin was seen to disorder the lysine sites of interaction on the surface of the protein and appeared to cause an extensive derangement of secondary structure, such that no stable $\alpha$-helices can exist in the protein backbone with a lifetime longer than around $10^{-6} \mathrm{~s}$. The results suggest that cytochrome $c$ reversibly denatures on complexation with cardiolipin bilayers, such that the electronic ground state prevailing in the native structure of both oxidized and reduced protein can convert to high-spin states with greater magnetic susceptibility. This direct observation of the molten globule in a membrane protein induced upon binding and may well be an important observation bearing relevance to protein insertion into membranes.

Phosphorus-31 NMR has been used to investigate the interaction of cytochrome $c$ with mixed lipid bilayers of the anionic lipids dioleoylphosphatidylglycerol, dioleoylphosphatidylserine, and diacylphosphatidylinositol [57]. All phosphorus-31 NMR spectra revealed the typical line shapes characteristic of phospholipids in liquid-crystalline bilayers. The effects on the phosphorus-31 chemical shift anisotropy (CSA) for each system reflect particular modes of phospholipid head-group interaction with cytochrome $c$. A distinct increase in the CSA for DOPS bilayers was observed upon binding of cytochrome $c$, which was thought to arise from a partial restriction of the amplitude of motion on this phospholipid head group. Phosphorus-31 NMR spin-lattice $\left(T_{1}\right)$ relaxation times of the various phospholipid-cytochrome $c$ complexes show that conformational changes occur in the protein on binding to anionic phospholipids. In common with the interaction with cardiolipin bilayers (see above), protein conformational changes are observed through paramagnetic enhancement of the measured phosphorus-31 spinlattice relaxation times for lipid phosphates as a result of the conformational changes in the protein in which the heme becomes accessible to lipid phosphate upon binding to charged bilayer surfaces. However, the phosphorus-31 $T_{1}$ values for the various complexes with cytochrome $c$ show a different temperature dependence for each lipid, revealing different modes of protein interaction for each of the different lipid head groups, The phosphate of phos-

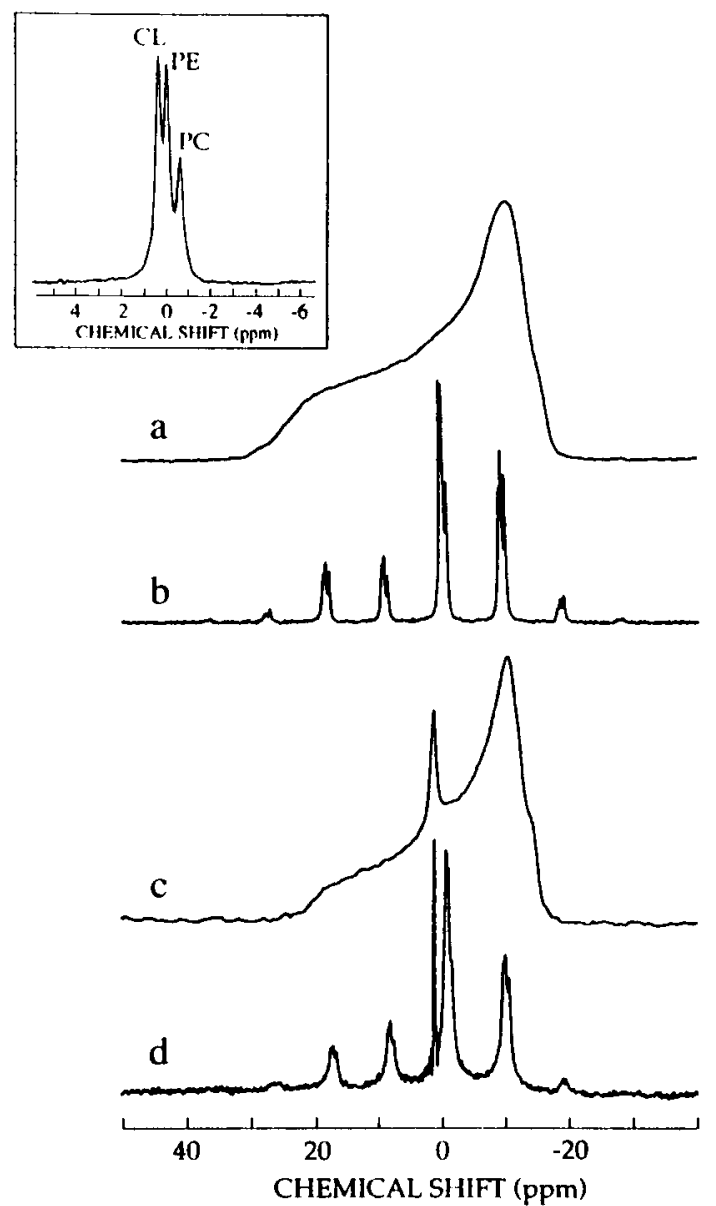

Fig. 11. Proton-decoupled, phosphorus-31 NMR spectra for a model of the mitochondrial membrane containing cardiolipin, phosphatidylethanolamine and phosphatidylcholine (1:2:2 molar ratio) without (a,b) and with (c,d) cytochrome $c$ (20:1 lipid/ protein molar ratio). Spectra from static samples (a,c) show that the protein induces a near-isotropic (narrow line) spectral component (c), and from the magic angle spinning approach $(\mathrm{b}, \mathrm{d})$, in which the three individual phospholipid components can be resolved (see inset), indicates that the cardiolipin is motionally restricted (from line broadening) upon interaction with cytochrome $c$, but it is the phosphatidylethanolamine which produces the near isotropic component. Adapted from Ref. [57].

phatidylserine was most efficiently relaxed by cytochrome $c$, while the relaxation of the phosphate in the phosphatidylinositol head group was not affected. These conclusions from the NMR experiments are supported by differential scanning calorimetry results, which indicated that the binding of cytochrome $c$ to the anionic phospholipid bilayers results in a loosening and/or destabilization of the 
overall protein structure. The phosphatidylserineand phosphatidylinositol-bound cytochrome $c$ shows the larger destabilization of the protein structure as revealed by the poor cooperativity of its thermally induced denaturation, while in the phosphatidylglycerol-cytochrome $c$ complexes the protein seems to preserve a more native-like structure.

To gain still further insight into the specificity of cytochrome $c$ interactions with individual lipids, a model of the inner mitochondrial membrane was constructed with dioleoylphosphatidylcholine, dioleoylphosphatidylethanolamine, and cardiolipin at a molar ratio of $2: 2: 1$, and the interaction of cytochrome $c$ with this mixed membrane investigated by static and MAS solid-state phosphorus-31 NMR [79,80] (Fig. 11). The static phosphorus-31 NMR spectrum of the three-component membrane is a typical broad powder pattern for phospholipids in a bilayer structure, and is a result of three overlapping spectra of each individual phospholipid component in the mixed membrane, with an average effective chemical shift anisotropy of approximately $41 \mathrm{ppm}$. Using MAS NMR methods, three resolved resonances are observed in the narrowed MAS phosphorus31 NMR spectrum, each of which has been assigned to each lipid component in the mixed membrane. This allows the investigation of individual phospholipid-protein interactions in multicomponent lipid bilayers. The interaction of cytochrome $c$ with each lipid in a model mitochondrial membrane could now be evaluated. Phosphorus-31 spin-lattice $\left(T_{1}\right)$ relaxation times for each lipid phosphate were measured as a function of temperature, in the absence and presence of bound cytochrome $c$. The $T_{1}$ values were not affected for any lipid upon binding of cytochrome $c$ over the temperature range analyzed. However, averaging of the phosphorus-31 chemical shift anisotropy for the cardiolipin component in the mixed bilayers at lower temperatures ceases to be axially symmetric on binding of cytochrome $c$, while for zwitterionic components the axial symmetry is retained over the temperature interval studied here. The results suggest that the strongest interaction of cytochrome $c$ in this model mitochondrial membrane occurs with the cardiolipin component, but less so with the other components. Some lipid lateral-phase separation in the mixed bilayer seems to be induced on binding of cytochrome $c$, in which phosphatidyl- ethanolamine is segregated into isotropic structures, as yet unresolved in molecular detail, on the phosphorus-31 NMR time scale.

\subsection{Type II myosin}

Myosin is an important biological motor in many systems, usually powered by the hydrolysis of ATP, and structural models have been proposed in both unicellular and organisms and animal cells. Singleheaded type I myosin has been shown to interact with membranes previously, but deuterium NMR has now been used for the first time to show the interaction of type II, conventional myosin to behave in a similar way to type I myosin [81]. Although no interaction was seen with zwitterionic membranes in bilayers, a direct electrostatic interaction was observed with charged (PG) bilayers and the tail of the protein which has a concentration of lysine residues. When ATP and $\mathrm{Mg}^{2+}$ were complexed to the head domain of myosin, a reduced interaction was observed, indicating that type II myosin can interact with membranes without the need for a specific hydrophobic domain or an anchor in the protein molecule, provided that charged lipids are present in the bilayer [81].

\section{Integral protein-lipid interactions}

In liquid-crystalline bilayers, no firm evidence exists to show that most integral proteins motionally constrain phospholipids on the time scale corresponding to the anisotropy averaging of the ${ }^{31} \mathrm{P}$ chemical shift or deuterium quadrupolar interactions. As stated above, titration of lipids and proteins in reconstituted bilayers are complex when protein associations or even irreversible aggregation, which can be more of a problem with integral rather than peripheral proteins, occurs in a concentration dependent way. Until reconstitution methods are more predictable, and the biochemistry of such complexes well defined, any biophysical method for studying lipid-protein interactions will not be simple. In some cases, however, spectroscopic information about such systems can be useful in defining protein-protein association behaviour itself. 


\subsection{Gramicidin}

An insight into the molecular interaction required for stability of an ion channel protein with a membrane has been obtained through studies of gramicidin interaction with dimyristoyl phosphatidylcholine bilayers using solid-state NMR and molecular dynamics simulations [82]. Most of the non-polar residues were observed to undergo isomerization transitions between multiple conformations. In contrast, the tryptophan side chains were seen to undergo fluctuations around a well-defined conformation stabilized by the membrane. Their hydrophobic moiety was buried in the bilayer hydrophobic core with the indole-NH group pointing towards to bulk solution, where it can make hydrogen bonds with the lipid glycerol backbone or with interfacial waters. Similar embedded tryptophans into lipid bilayers has been observed in other peptides, and may also be a common feature which contributes to the stability of many other integral membrane proteins.

Such detailed molecular associations confirm structure predictions of membrane proteins. The comparison between experiment and theory showed that the total interaction energy between a lipid and gramicidin, extends over a very large range from 0 $-50 \mathrm{kcal} / \mathrm{mol}$, from exclusively van der Waals interactions to nearly equal contributions from van der Waals and electrostatic terms. The most energetically favourable interactions were short lived, in contrast with boundary lipid models of lipids interacting with much larger proteins; the diffusion energies for small single helix peptides and a lipid are comparable, so this observation is probably not too surprising but does highlight the problem with comparing peptides and large proteins in describing lipid-protein interactions.

\subsection{Bacteriophage M13 coat protein}

The way in which the M13 coat protein interacts with lipids in bilayers has been extensively studied by a range of methods $[83,84]$. Following non-pathogenic infestation of Escherichia coli, the M13 coat protein is assembled in the outer membrane in anticipation of the gene component to be extruded from the bacterium, and assembly of about 2700 M13 coat protein units around a central core to form the ma- ture bacteriophage. In lipid model membranes, the protein can adopt two conformations, a predominantly $\alpha$-helical and a $\beta$-polymeric form, that differ in their aggregation characteristics, the latter form being artificial and only found in reconstituted bilayers. Protein aggregation changes with the lipidprotein ratio and the type of lipid in the bilayers. Studies of the self-association of the $\alpha$-form of the protein are, therefore, functionally relevant since the coat protein assembles in the E. coli. plasma membrane before maturation and release from the bacterium $[83,84]$.

The effect of incorporation of various amounts of M13 bacteriophage coat protein on the bilayer order and acyl chain motion in dimyristoylphosphatidylcholine bilayers was investigated using deuterium NMR of specifically deuterated palmitic acid as a bilayer probe, phosphorus NMR and additional spin-label electron spin resonance (ESR) [85]. The secondary structure of the M13 coat protein in these bilayers was determined from circular dichroism spectra and indicated that the protein conformation is predominantly a $\beta$-structure (more than $75 \%$ ). Although the amounts of M13 coat protein in the bilayer did not affect the order of the deuterium-labelled positions, the spin-spin $\left(T_{1}\right)$ relaxation times decreased at higher protein levels, especially for the carbon in the centre of the bilayer. The spin-label ESR spectra of the same system show a second, motionally restricted spectral component, that is not observed by deuterium NMR. The NMR and ESR results are consistent with a model in which the fatty acid molecules are in a fast two-site exchange $\left(v_{\mathrm{ex}} \sim 10^{7} \mathrm{~s}^{-1}\right)$ between the sites in the bulk of the lipid bilayer and the motionally restricted sites on the coat protein.

In a similar study, in which the head group of the phosphatidylcholine was deuterated in the trimethyl segments of the choline head group, two-component deuterium and phosphorus-31 NMR spectra have been observed from bilayer complexes containing the coat protein, indicating slow exchange (on the deuterium quadrupole anisotropy and phosphorus31 chemical shift averaging time scales) of lipid molecules of $<10-3 \mathrm{~Hz}$ between two motionally distinct environments in the complexes [86]. The fraction of the isotropic spectral component for both deuterium and phosphorus nuclei, increases with in- 


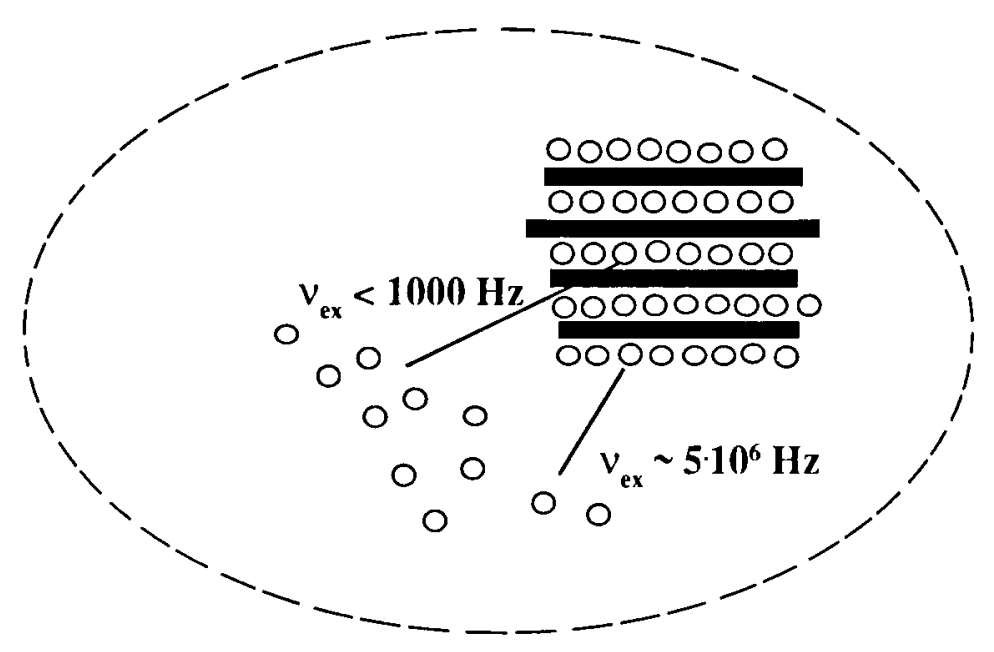

Linear aggregate of M13 coat protein. Lipid molecule.

Fig. 12. A suggested arrangement of the bacteriophage M13 coat protein in bilayers, as deduced from both nitroxide spin-label ESR and deuterium-NMR studies. The 'trapped' lipid observed deuterium NMR studies (from head group deuterated lipids), are also observed in the spin-label studies along with the 'boundary' lipids near the aggregates. A linear protein array was suggested from quantification of the lipid-protein stoichiometry deduced from a slow, two-site exchange model for the protein-lipid interaction [15]. A similar arrangement may occur in assembly of the bacteriophage on emergence from the $E$. coli host following non-pathogenic infestation $[83,84,111]$.

creasing M13 protein concentration, and this component is attributed to lipid head groups which are disordered relative to their order in protein-free bilayers. The chemical exchange rate of lipid molecules between the two motionally distinct environments has been estimated to be $20-50 \mathrm{~Hz}$ by steady-state line-shape simulations of the deuterium spectra from the complexes using exchange-coupled modified Bloch equations. The off-rate was, as expected from one-to-one exchange, independent of the $\mathrm{L} / \mathrm{P}$ ratio $\left(\tau_{\text {off }}^{-1}=0.23 \mathrm{~s}^{-1}\right)$. It was suggested that the proteinassociated lipid may be trapped between closely packed parallel aggregates of M13 coat protein and that the high local concentration of protein in a onedimensional arrangement in lipid bilayers may be required for the fast reassembly of phage particles before release from an infected cell (Fig. 12).

The interaction of the M13 bacteriophage major coat protein in the $\alpha$-oligomeric form, with specifically deuterated phospholipid head groups which mimic the E. coli inner membrane, has been studied using NMR methods [87]. Here, the coat protein does not give rise to trapped lipids as observed with M13 coat protein in the $\beta$-polymeric form
[86]. The quadrupolar splittings of the alpha headgroup methylene deuterons of deuterated phosphatidylcholine and phosphatidylethanolamine decrease, whereas the quadrupolar splittings of the $\beta$ headgroup methylene deuterons of the two lipids increase with increasing protein content. All deuterated segments in the phosphatidylglycerol head group show the same relative decrease of the NMR quadrupolar splittings. These results are interpreted in terms of a change in torsion angles of the methylene groups, induced by positive charges, probably lysine residues of the protein at the membrane surface. It is concluded that there is no strong specific interaction between one of the lipid types examined and the M13 coat protein. From the spin-spin $\left(T_{2 \mathrm{e}}\right)$ relaxation time and spin-lattice $\left(T_{1 z}\right)$ relaxation time of all deuterated lipids, it is concluded that at the bilayer surface only slow motions are affected by the M13 coat protein.

A comparison has also been made of the interaction of the coat protein of bacteriophage M13 in a predominant $\alpha$-helix conformation and in a predominant $\beta$-sheet conformation [88], using deuterium and phosphorus-31 NMR. From the deuterium NMR 
studies on specific head group and chain deuterium labelled phospholipids, it was found that the protein in the predominant $\beta$-sheet conformation causes a fraction of lipids to be trapped. By combining the results from the head group and acyl chains of the phospholipids, it is concluded that the trapped lipids are arranged in a non-bilayer structure, probably caused by a misfitting of the hydrophobic core of the protein and the membrane bilayer. The protein in the predominant $\alpha$-helix conformation fits well in the lipid bilayer, and has only minor influences on the surrounding lipid matrix. A model was proposed to explain the presence of the trapped lipid in the lipid/protein systems.

\subsection{Glycophorin A}

Rotational resonance (RR) NMR, circular dichroism (CD), and attenuated total reflection Fourier transform infrared (ATR-FTIR) spectroscopy have been used to establish the secondary structure and orientation of peptides corresponding to the transmembrane domain of human glycophorin $\mathrm{A}$ in dimyristoylphosphatidylcholine bilayers [89]. The peptide was shown to be largely $\alpha$-helical and generally perpendicular to the bilayer plane. High-resolution structural data on the glycophorin A transmembrane peptide were obtained by measuring the rate of magnetization exchange between pairs of specific ${ }^{13} \mathrm{C}$ labels using solid-state, rotational resonance NMR, to give distances between the spin-pairs, with a resolution on the order of $0.3 \AA$. Distances of $4.5 \AA$ between $i$ and $i+1$ residues in the transmembrane part of the protein confirm a helical peptide geometry. However, longer distances are observed between residues at the polar-apolar membrane interface, which suggests that the transmembrane helix unravels at the membrane interface.

Rotational resonance (RR) NMR methods have been used to determine interatomic distances between specific ${ }^{13} \mathrm{C}$ labels incorporated into lipids in a bilayer and a hydrophobic peptide corresponding to the trans-membrane domain of human glycophorin A [90]. Intermolecular magnetization exchange rates have been measured between the phosphatidylcholine chain and the ${ }^{13} \mathrm{COH}$ position of $\mathrm{Y}-93$ in the glycophorin transmembrane domain. These intermolecular distance measurements demonstrate that the relative orientation and location of membrane lipids and peptides can be established using solid-state rotational resonance NMR methods, giving resolution about lipid-protein interactions at crystallographic resolution. These results demonstrate that high-resolution measurements of local protein structure, and the effects of the membrane on that protein structure, can be made in lipid bilayers, giving a new approach to molecular detail of lipid-protein interactions.

\subsection{Proteolipid apoprotein}

In a number of studies, it has been shown that the spectral line shapes in deuterium NMR are sensitive to lipid-protein interactions [29]. A line-shape analysis of the relaxation mechanisms has been carried out in a study of the association in bilayers of acyl chain deuterated phosphatidylcholine with the integral proteolipid apoprotein (PLP) from bovine myelin [91]. All the spectra obtained for fluid phase bilayers were shown by simulation to consist of a single-component, and displayed single-component relaxation time, indicating either a homogeneous lipid environment or rapid exchange through lateral diffusion between environments of differing lipid mobility. The results show that exchange between these component populations must be fast enough to give averaging of the quadrupolar anisotropy and relaxation rates.

The influence of the myelin-proteolipid apoprotein (PLP) on the order of the acyl chains of dimyristoyl phosphatidylcholine specifically deuterated at three different positions (C6, C13, and $\mathrm{C} 14)$ in the $s n-2$ acyl chain has been studied in reconstituted systems [91]. Data from quadrupolar echo line shapes and saturation recovery experiments were fitted by numerical solution of the stochastic Liouville equation, using a model that includes both inter- and intramolecular motions of the lipids [92]. In this way, an assignment of the various motional modes of the lipid segments was made. In the fluid phase, the protein has little influence on either the chain order or the population of gauche rotational isomers, but strongly retards the chain dynamics. At $35^{\circ} \mathrm{C}$, the presence of the protein is to increase the correlation time for various chain motions; chain fluctuations increased from $20 \mathrm{~ns}$ to $650 \mathrm{~ns}$, chain rotation from $10 \mathrm{~ns}$ to $180 \mathrm{~ns}$, and the gauche isomer lifetime along 
the chain increased from $0.15 \mathrm{~ns}$ to $1.75 \mathrm{~ns}$ for pure lipid bilayers and for a recombinant of protein/lipid ratio $0.075 \mathrm{~mol} / \mathrm{mol}$, respectively.

\subsection{Rhodopsin and band 3}

Polar head groups of phospholipids undergo rapid, large-amplitude motion at the bilayer surface. Since the axis of motional averaging is not known, probably being close to the 'magic angle' and hence giving small quadrupole splittings, experimentally determined values of the deuterium qudrupole splittings are sensitive to both amplitude of motion and the orientation of the whole polar group. It has been shown that this part of the bilayer is sensitive to both lipid-lipid interactions $[64,65]$ and protein-lipid associations $[29,30,73,93]$.

Fast exchange of lipids between the bulk phase and the protein-lipid interface has been demonstrated by deuterium NMR studies of the surface of bilayer membranes containing bovine retinal rhodopsin [93] and the human erythrocyte, anion-transport protein, band 3 [73]. Despite some spectral broadening (Fig. 2), indicative of changes in the deuterium spin-spin relaxation time, $T_{2}$, the experimentally estimated quadrupole splittings for head grouplabelled phosphatidylcholine were determined for a wide range (15 between $350: 1$ and 65:1, lipid/protein ratio) of lipid-to-protein ratios. In all cases a linear dependence of the observed quadrupole splittings with protein to lipid ratio was demonstrated and given as evidence for fast $\left(v_{\mathrm{ex}}<10^{4} \mathrm{~s}^{-1}\right)$ lipid-protein exchange on the deuterium NMR time scale for averaging of the quadrupolar interaction.

The state of band 3 aggregation, which has been shown by fluorescent labelling techniques to be temperature sensitive [94] has also been observed by deuterium NMR [73]. Since this aggregation is observed in both erythrocytes and phosphatidylcholine bilayers, it appears to be protein-, and not lipid-induced. In addition, the NMR studies demonstrated that protein-lipid interactions in this region of the bilayer (and possibly in the hydrophobic core also), when averaged throughout the whole membrane, are significantly reduced when the protein exists as large oligomers rather than as smaller units. The propensity for a number of proteins, for example, cytochrome $c$ oxidase, to aggregate upon reconstitution may in part explain the rather small protein-lipid interactions observed so far by NMR $[29,72]$. Clearly the number of lipids with access to an interface with a protein is significantly reduced if protein-protein contacts occur.

In oriented rod outer segment membranes, in which rhodopsin constitutes $\sim 95 \%$ of their total integral membrane protein, the phosphorus-31 chemical shift anisotropy is reduced almost to zero [95]. This behaviour is in contrast to the same protein when reconstituted into synthetic lipid bilayers, where at similar lipid-protein content, the choline head groups indicate a well oriented bilayer. This difference is not well understood, but the protein does perturb the lipid head-group structure, possibly as a result of protein surface disorder, in the heterogeneous natural membrane. Any matching of the bilayer to the protein hydrophobic dimension [1], may involve curvature of the bilayer in the natural membrane, which, through rapid lipid diffusion rates $\left(v_{\mathrm{ex}} \sim 10^{8} \mathrm{~s}^{-1}\right)$ [40], would average out the phosphorus-31 chemical shift anisotropy and implies a different packing constraint for the reconstituted protein. Indeed, in synthetic bilayers, rhodopsin can be locked into one of the two functionally significant conformers, the meta II form, as a result of the lipid interaction with the protein interface [96].

\subsection{Bacteriorhodopsin}

The effect of bacteriorhodopsin on the orientation of the head group of dimyristoylphosphatidylcholine in bilayers has been studied by phosphorus-31- and deuterium NMR [97]. Bacteriorhodopsin (bR), from the halophilic bacterium, Halobacterium salinarium, has been separated from the endogenous purple membrane lipids and reconstituted by detergent dialysis into bilayers dimyristoylphosphatidylcholine which was selectively deuterated at the choline head group. Complexes containing lipid/protein at molar ratios of $67: 1$ to $222: 1$ were produced under conditions to promote formation of large vesicles (mean diameters $600-700 \mathrm{~nm}$ ). The magnitudes of the deuterium-NMR quadrupole splittings recorded from the deuterium-labelled head-group segments, and the phosphorus-31-NMR chemical shift anisotropy (CSA) of the phosphate group varied linearly with the protein content in the complexes over the range 
of complexes studied. On increasing the proportion of bR in the DMPC-BR complexes, the deuteriumNMR quadrupole splittings measured from the choline gamma-methyl groups and the $\beta$-methylene segments and the phosphorus-31-NMR CSA increased in magnitude, while the deuterium-NMR quadrupole splitting from the $\alpha$-methylene segment decreased. Such opposing changes in the choline $\alpha$ - and $\beta$-methylene segment quadrupole splittings are similar to those reported on increasing the proportion of positively charged amphiphile at the bilayer surface [98]. It is suggested that $\mathrm{bR}$ presents a net positive charge to the phosphocholine head groups at the protein/ lipid interface.

\subsection{Membrane spanning domain of tyrosine kinase}

The receptor tyrosine kinase encoded by the neu/ erbB-2 proto-oncogene is constitutively activated by a single valine to glutamic acid substitution at position 664 in the predicted membrane-spanning sequence of the receptor. The structural changes involved in receptor activation with polarized FTIR and solid-state, MAS NMR spectroscopy have been explored [99]. The hydrophobic transmembrane sequence folds into a well-defined $\alpha$-helical structure spanning the membrane bilayer. Measurements of the $\mathrm{p} K_{\mathrm{a}}$ and ${ }^{13} \mathrm{C}$ chemical shift anisotropy of Glu 664 reveal that the side-chain carboxyl group is protonated and strongly hydrogen bonded when in the bilayer core. These studies provide direct evidence for glutamate hydrogen-bonding interactions in the mechanism of receptor dimerization and activation for membrane embedded protein, and show the importance of studying protein-protein associations in membranes, since they do not appear to occur in solution.

\subsection{Natural membranes}

In one of the few studies of lipid-protein interactions in natural membranes by solid-state NMR, deuterium NMR has been used to study the plasma membrane of a fatty acid auxotroph of $E$. coli grown on palmitic and oleic acids labelled at a number of sites in the chain. The apparent order parameter profile of the palmitoyl chain in the natural and proteinfree 1-palmitoyl-2-oleoyl phosphatidylcholine bi- layers was similar [100]. A direct comparison between order in a phosphatidylcholine bilayer and the natural, mixed-lipid membrane may not be valid, but essentially the same phenomenon was subsequently confirmed in determinations of the order parameters for $\mathrm{CD}$ bonds adjacent to the site of unsaturation in elaidic acid in liposomes of the total lipid extract (PE, $80 \mathrm{~mol} \%$; PG, 20 mole, with $93 \%$ of the lipid having 9,10-deuterated elaidic acid) and in both the inner and outer plasma membranes [101]. Only a $10-20 \%$ decrease in order parameters was observed in the natural membranes relative to the membrane derived liposomes. These studies suggest that the perturbation and decrease in intrinsic acyl chain order within a lipid bilayer by a protein is small and is most probably caused by the rough, invaginated protein interface presented to the lipid chains by the hydrophobic amino acid side groups [102].

More recently, the effect of cholesterol and lanosterol on the structure and dynamics of the cell membrane of Mycoplasma capricolum, a natural fatty acid autotroph that requires sterols for growth, has been studied by deuterium NMR [103]. Palmitic acid, labelled at $\mathrm{C} 7$ and $\mathrm{C} 8$ with deuterons, was added to the growth medium to report on the structure and dynamics of the plasma membrane, which is predominately phosphatidylglycerol (PG) $(60 \%)$ and cardiolipin (CL) $(35 \%)$. The incorporation of cholesterol and the ratio of saturated to unsaturated fatty acids increased non-linearly with increases in exogenous cholesterol level, whereas the levels of phospholipid increased only slightly. At the growth temperature of $37^{\circ} \mathrm{C}$, the residual deuterium quadrupole splittings for the labelled lipids in the natural membranes were found to be similar to those found in cholesterol/lecithin binary dispersions of similar cholesterol content to the natural membrane. However, the deuterium spin-spin relaxation times $\left(T_{2 \mathrm{e}}\right)$, indicative of slow ( $\mu \mathrm{s}-\mathrm{ms})$ molecular motions in the lipids, were independent of cholesterol content in the natural membranes, and considerably shorter $(170 \pm 10 \mu \mathrm{s})$ than when measure in lipid extracts $(320-420 \mu \mathrm{s})$ from the natural membranes. It was concluded that lipid-protein interactions in the cell membrane is the dominant mechanism responsible for the reduced $T_{2 \mathrm{e}}$. In addition, evidence was found for the coexistence of gel and liquid-crystalline states in cells having low cholesterol levels. Lanosterol appeared to 
be less effective than cholesterol in modulating cell membrane physical properties, as detected by deuterium quadrupole splittings and relaxation times, and the cell membranes were found to have a similar $T_{2 \mathrm{e}}$ regardless of the growth conditions or sterols added.

\section{Future possibilities}

With some hope of crystal structures of membrane proteins now becoming realized, and the powerful solid-state NMR methods refined to such a degree that distance measurements at atomic resolution within membranes of proteins and lipids is being achieved [31-33], it seems likely that it is going to be possible to identify specific lipid-protein associations, even to which lipids moieties and which protein residues are involved in such interactions. Spectroscopic methods in general, can identify the dynamic nature of any lipid-protein associations. Eventually all such structural and spectroscopic information does need to be related to function, and the way in which phospholipids and lipids modulate and regulate membrane function is still some way off.

Asked for a personal, but not comprehensive 'wish list' of information that needs be resolved about lipid-protein interactions, some of which is being currently addressed, one might include:

1. How are integral membranes sealed effectively in a membrane [104]?

2. If there is a specific interaction of some lipids with some proteins, what are the residues and parts of the lipid involved, and how? (solid-state NMR is beginning to answer this question in some systems)

3. Can membrane lipids percolate into the packed helices of integral proteins and modulate packing and hence function?

4. Are interfacial regions of membranes between laterally distinct domains, regions through which proteins (and perhaps other polar solutes) can translocate [105]?

5. Can membrane 'impurities', such as drugs, anaesthetics and other lipophiles, concentrate at the protein-lipid interface and thereby modulate protein function as a result of penetration into the protein hydrophobic components and perturbation of protein structure directly $[16,106]$ ?

6. Are the slow director fluctuations observed in membranes and induced by proteins [72,75,102] important functionally?

7. Do some lipids have special roles to play, at the molecular level, in membrane stability and function - for example, is phosphatidylethanolamine a 'special' lipid in view of its capacity to form relatively strong hydrogen bonds without water [65], and how and why is cardiolipin peculiar to electron transporting membranes [107]?

8. What contribution do the aromatic residue-lipid interactions, at the membrane polar-apolar interface, make to protein stability and function [108$110]$ ?

Some determined and detailed attempts are being made to address many of these topics (see other contributions in this issue), and until more is known at the detailed structural and dynamic level from a greater body of information gained through studies of protein-lipid interactions, we can only speculate on some of these points, and generalizations may never actually be possible, anyway, for heterogeneous, natural biomembranes. Solid-state NMR methods do have a contribution to make, and will be just one weapon in the arsenal of tools which will ultimately give us the insight into protein lipid interaction which is required to relate structure to function in such complex systems.

\section{Acknowledgements}

A.W. is a BBSRC Senior Research Fellow, and their support is gratefully acknowledged.

\section{References}

[1] O.G. Mouritsen, Curr. Opin. Colloid Interface Sci. 3 (1998) 78-87.

[2] H. Sandermann, T.M. Duncan, J.O. McIntyre, S. Fleischer, in: A. Watts (Ed.), Protein-Lipid Interactions, Elsevier, 1993, pp. 67-86.

[3] S.E. Rankin, D.E. Raines, L.A. Dalton, K.W. Miller, in: A. Watts (Ed.), Protein-Lipid Interactions, Elsevier, 1993, pp. 213-230. 
[4] D.D. Thomas, J.E. Mahaney, in: A. Watts (Ed.), ProteinLipid Interactions, Elsevier, 1993, pp. 301-320.

[5] F.J. Barrantes, in: A. Watts (Ed.), Protein-Lipid Interactions, Elsevier, 1993, pp. 231-258.

[6] K.M. Merz, B. Roux, Biological Membranes. A Molecular Perspective for Computation and Experiment, Birkhäuser, Boston, 1996.

[7] D. Marsh, in: A. Watts (Ed.), Protein-Lipid Interactions, Elsevier, 1993, pp. 41-66.

[8] M.B. Sankaram, D. Marsh, in: A. Watts (Ed.), Protein-Lipid Interactions, Elsevier, 1993, pp. 127-162.

[9] J.R. Arrondo, F.M. Goni, in: A. Watts (Ed.), Protein-Lipid Interactions, Elsevier, 1993, pp. 321-350.

[10] C.E. Castuma, M. Lamy-Freund, R.R. Brenner, S. Schreir, in: A. Watts (Ed.), Protein-Lipid Interactions, Elsevier, 1993, pp. 87-106.

[11] T.P. McGee, M.K.Y. Fung, V.A. Bankaitis, in: A. Watts (Ed.), Protein-Lipid Interactions, Elsevier, 1993, pp. 163 190.

[12] S.H. White, Membrane Protein Structure, OUP, New York, 1994.

[13] S.H. White, M.C. Wiener, in: K.M. Merz, B. Roux (Eds.), Biological Membranes. A Molecular Perspective for Computation and Experiment, Birkhäuser, Boston, 1996, pp. 127144.

[14] G. Ceve, Biochemistry 26 (1987) 6350.

[15] A. Watts, L.C.M. van Gorkom, in: P. Yeagle (Ed.), The Structure of Biological Membranes, CRC Press, New York, 1991, pp. 307-377.

[16] D. Fraser, S. Louro, L.I. Horvath, K. Miller, A. Watts, Biochemistry 29 (1990) 2664-2669.

[17] A. Watts, J.J.H.H.M. de Pont, Progress in Protein-lipid Interactions vol. 1, Elsevier, Amsterdam, 1985.

[18] A. Watts, J.J.H.H.M. de Pont, Progress in Protein-lipid Interactions vol. 2, Elsevier, Amsterdam, 1986.

[19] A. Watts, Protein-Lipid Interactions, New Comprehensive Biochemistry Series Vol. 25, Elsevier, Amsterdam, 1993.

[20] A. Watts, in: J.A.F. Op den Kamp (Ed.), Biological Membranes: Structure, Biogenesis and Dynamics, NATO ASI Series, H82, 1994, pp. 79-92.

[21] A. Watts, in: G. Cevc (Ed.), Phospholipids Handbook, Marcel Dekker, New York, 1993, pp. 687-740.

[22] A. Watts, in: R. Glazer, D. Gingell (Eds.), Biophysics of the Cell Surface, Springer, Berlin, 1990, pp. 23-50

[23] A. Watts, Studia Biophys. 127 (1988) 29-36.

[24] A. Watts, in: S. Harding, A.J. Rowe (Eds.), Dynamic Properties of Biomolecular Assemblies, Royal Chemical Society, London, 1988, pp. 320-347.

[25] A. Watts, in: K.A.W. Wirtz (Ed.), Membrane Receptors, Dynamics, and Energetics, NATO ASI Series, Plenum Press, New York, 1987, pp. 329-340.

[26] A. Watts, J. Bioenerg. Biomembr. 19 (1987) 625-653.

[27] A. Watts, Bull. Mag. Res. 9 (1987) 66-70.

[28] J. Seelig, A. Seelig, L. Tamm, in: P.C. Jost + O. Hayes Griffith (Ed.) Lipid-Protein Interactions, vol. 2, John Wiley, New York, 1982, pp. 127-148.
[29] D. Marsh, A. Watts, in: R. Aloia (Ed.), Recent Advances in Membrane Fluidity, vol. 4, Alan R. Liss, New York, 1988, pp. 163-200.

[30] A. Watts, F. Sixl, N.J.P. Ryba, C.E. Dempsey, P.J. Brophy, in: Govil, Khetrapal, Saran (Eds.), Magnetic Resonance in Biology and Medicine, 1985, Academic Press, pp. 349-361.

[31] A. Watts, A.S. Ulrich, D.A. Middleton, Mol. Membr. Biol. 12 (1995) 233-246.

[32] S.O. Smith, K. Ascheim, M. Groesbeek, Q. Rev. Biophys. 29 (1996) 395-449.

[33] A. Watts, P.J.R. Spooner, D.M. Middleton, G. Gröbner, P.T.F. Williamson, Eur. Biophys. J. (1998) in press.

[34] D. Marsh, A. Watts, in: Colowick, Kaplan (Eds.), Methods in Enzymology vol. 88, Academic Press, New York, 1982, pp. $762-772$.

[35] D. Marsh, A. Watts, in: P.C. Jost, O. Hayes Griffith (Eds.), Lipid-protein Interactions vol. 2, Wiley-Interscience, New York, 1982, pp. 53-126.

[36] A. Watts, Nature 294 (1981) 512.

[37] J. Seelig, Q. Rev. Biophys. 10 (1977) 353-418.

[38] J. Seelig, Biochim. Biophys. Acta 939 (1978) 267.

[39] J. Seelig, A. Seelig, Q. Rev. Biophys. 13 (1980) 9-61.

[40] R. Clegg, W. Vaz, in: A. Watts, J.J.H.H.M. de Pont (Eds.), Progress in Protein-lipid Interactions vol. 1, Elsevier, Amsterdam, 1982, pp. 173-230.

[41] Z.-y. Peng, Z.Y. Eng, N. Tjandra, V. Simplaceau, C. Ho, Biophys. J. 56 (1989) 877-886.

[42] Z.-Y. Peng, V. Simplaceanu, I.J. Lowe, C. Ho, Biophys. J. 54 (1988) 81-95.

[43] O.B. Peersen, E.A. Pratt, H.T.N. Truong, C. Ho, G.S. Rule, Biochemistry 29 (1990) 3256-3262.

[44] C. Glaubitz, A. Watts, J. Mag. Res. 130(2) (1998), pp. 305316.

[45] M. Auger, Biophys. Chem. 68 (1997) 233-241.

[46] S.J. Opella, Nature Struct. Biol. NMR supplement, Oct. (1997) 845-849.

[47] B. de Kruijff, P.R. Cullis, A.H. Verkleij, M.J. Hope, C.J.A. van Echteld, T.F. Taraschi, P. Van Hoogevest, J.A. Killian, A. Rietveld, A.T.M. Van der Steen, in: A. Watts, J.J.H.H.M. de Pont (Eds.), Progress in Protein-lipid Interactions, vol. 1, Elsevier, Amsterdam, 1985, Chapter 3.

[48] A.G. Lee, J.M. East, in: A. Watts (Ed.), Protein-Lipid Interactions, Elsevier, 1993.

[49] E. Racker, Reconstitution of Transporters, Receptors and Pathological States, Academic Press, New York, 1985.

[50] A.I.P.M. de Kroon, J. de Gier, B. de Kruijff, in: A. Watts (Ed.), Protein-lipid interactions, Elsevier, 1993, pp. 107126.

[51] B.A. Seaton, M.F. Roberts, in: K.M. Merz, B. Roux (Eds.), Biological Membranes. A Molecular Perspective for Computation and Experiment, Birkhäuser, Boston, 1996, pp. 355404.

[52] D. Murray, N. Ben-Tal, B. Honig, S. McLaughlin, Structure (Lond.) 5, (8) (1997) 985-989.

[53] M.C. Sabra, O.G. Mouritsen, Biophys. J. 74 (1998) 745-752.

[54] F. Sixl, A. Watts, Biochemistry 24, (27) (1985) 7906-7910. 
[55] M.A. Carbone, P.M. Macdonald, Biocehmistry 35 (1996) 3368-3378.

[56] C.E. Dempsey, A. Watts, Biochemistry 26 (1987) 58035811.

[57] T.J.T. Pinheiro, A. Watts, Biochemistry 33 (1994) 2459 2467.

[58] G. Gröbner, A. Watts, Biophys. J. (1998) submitted.

[59] M.H. Biaggi, T.J.T. Pinheiro, A. Watts, M.T. Lamy-Freund, Eur. Biophys. J. 24, (4) (1996) 251-259.

[60] P.L. Yeagle, A.R. Dentino, F.T. Smith, P.J.R. Spooner, A. Watts, Biochemistry 32, (45) (1993) 12197-12202.

[61] E.J. Dufourc, I.C.P. Smith, J. Dufourcq, Biochemistry 25 (1986) 6448-6455.

[62] H. Akutsu, J. Seelig, Biochemistry 20 (1981) 7366-7373.

[63] C.E. Dempsey, M. Bitbol, A. Watts, Biochemistry 28 (1989) 6590-6596.

[64] F. Sixl, A. Watts, Biochemistry 24 (1982) 6446-6452.

[65] F. Sixl, A. Watts, Proc. Natl. Acad. Sci. U.S.A. 80 (1983) $1613-1615$.

[66] M. Roux, J.M. Neumann, R.J. Hodges, P.F. Devaux, M. Bloom, Biochemistry 28 (1989) 2313-2321.

[67] B. Sankaram, Biophys. J. 67 (1994) 105-112.

[68] R.E. Koeppe, J.A. Killian, T.C. Vogt, B. de Kruijff, M.J. Taylor, G.L. Mattice, D.V. Greathouse, Biochemistry 34, (29) (1995) 9299-9306.

[69] F. Sixl, P.J. Brophy, A. Watts, Biochemistry 23 (1984) 2032 2039.

[70] E. London, G.W. Feigenison, Biochemistry 20 (1981) 1939 1948.

[71] J.M. East, A.J. Lee, Biochemistry 21 (1982) 4144-4151.

[72] M.R. Paddy, F.W. Dahlquist, J.H. Davis, M. Bloom, Biochemistry 20 (1981) 3152-3162.

[73] C.E. Dempsey, N.J.P. Ryba, A. Watts, Biochemistry 25 (1986) 2180-2187.

[74] M. Hayer-Hartl, P.J. Brophy, D. Marsh, A. Watts, Biochemistry 32 (1993) 9709-9713.

[75] P.J.R. Spooner, A.A. Duralski, S.E. Rankin, T.J.P. Pinheiro, A. Watts, Biophys. J. 65 (1993) 106-112.

[76] P.J.R. Spooner, A. Watts, Biochemistry 30 (1991) 38713879.

[77] P.J.R. Spooner, A. Watts, Biochemistry 30 (1991) 3880 3885.

[78] P.J.R. Spooner, A. Watts, Biochemistry 31 (1992) 10129 10138.

[79] T.J.T. Pinheiro, A. Watts, Biochemistry 33 (1994) 24512458.

[80] T.J.T. Pinheiro, A.A. Duralski, A. Watts, Biochemistry 33 (1994) 4896-4902.

[81] J.A.G. Areas, G. Gröbner, C. Glaubitz, A. Watts, Biochemistry 37 (1998) 5582-5588.

[82] T.B. Woolf, B. Roux, Proc. Natl. Acad. Sci. U.S.A. 91 (1994) 11631-11635.

[83] M.A. Hemminga, J.C. Sanders, C.J.A.M. Wolfs, R.B. Spruijt, in: A. Watts (Ed.), Protein-Lipid Interactions, New Comprehensive Biochemistry, vol. 25, Elsevier, 1993, pp. 191-212.
[84] M.A. Hemminga, J.C. Sanders, R.B. Spruijt, in: H. Sprecher (Ed.), Progress in Lipid Research, vol. 31, 1992, pp. 301-333.

[85] K.P. Datema, R.B. Spruijt, C.J. Wolfs, M.A. Hemminga, Biochim. Biophys. Acta 944, (3) (1988) 507-515.

[86] L.C.M. Van Gorkom, L.I. Horvath, M.A. Hemminga, B. Sternberg, A. Watts, Biochemistry 29, (16) (1990) 3828 3834.

[87] J.C. Sanders, T.W. Poile, R.B. Spruijt, N.A.J. Van Nuland, A. Watts, M.A. Hemminga, Biochim. Biophys. Acta 1066, (1) (1991) 102-108.

[88] J.C. Sanders, T.W. Poile, C.J.A.M. Wolfs, M.A. Hemminga, Biochim. Biophys. Acta 1110, (2) (1992) 218-224.

[89] S.O. Smith, J. Hamilton, A. Salmon, B.J. Bormann, Biochemistry 33 (1994) 6327-6333.

[90] S.O. Smith, R. Jonas, M. Braiman, B.J. Bormann, Biochemistry 33 (1994) 6334-6341.

[91] P. Meier, J.-H. Sachse, P.J. Brophy, D. Marsh, G. Kothe, Proc. Natl. Acad. Sci. U.S.A. 84 (1987) 3704-3708.

[92] P. Meier, E. Ohmes, G. Kothe, J. Chem. Phys. 85 (1986) 3598-3614.

[93] N.J.P. Ryba, C.E. Dempsey, A. Watts, Biochemistry 25 (1986) 4818-4825.

[94] T. Mühlebach, R.J. Cherry, Biochemistry 24 (1985) 975983.

[95] G. Gröbner, A. Taylor, P.T.F. Williamson, G. Choi, C. Glaubitz, J.A. Watts, W.J. de Grip, A. Watts, Anal. Biochem. 254 (1997) 132-136.

[96] G. Gröbner, G. Choi, I. Burnett, C. Glaubitz, A. Wattts, FEBS Lett. 422 (1998) 201-204.

[97] P. Gale, A. Watts, Biochim. Biophys. Acta 1106, (2) (1992) 317-324.

[98] J. Seelig, P.M. Macdonald, P.G. Scherer, Biochemistry 26 (1987) 7535-7541.

[99] S.O. Smith, C.S. Smith, B.J. Bormann, Nature Struct. Biol. 3, (3) (1996) 252-258.

[100] H.U. Gally, G. Pluschke, P. Overath, J. Seelig, Biochemistry 18 (1979) 5605-5610.

[101] H.U. Gally, G. Pluschke, P. Overath, J. Seelig, Biochemistry 20 (1981) 5605-5610.

[102] M. Bloom, Can. J. Phys. 57 (1979) 2227-2230.

[103] T.H. Huang, A.J. Desiervo, Q.X. Yang, Biophys. J. 59 (1991) 691-702.

[104] W.C. Wimley, S.H. White, Nature Struct. Biol. 3, (10) (1996) 842-848.

[105] W.L.C. Vaz, F.F. Paulo, P.F.F. Almeida, Curr. Opin. Struct. Biol. 3 (1993) 482-488.

[106] A. Watts, Ann. N.Y. Acad. Sci. 625 (1991) 653-669.

[107] P.F. Knowles, A. Watts, D. Marsh, Biochemistry 18 (1979) 4480-4487.

[108] C. Gray, S.A. Tatulian, S.A. Wharton, L.K. Tamm, Biophys. J. 70, (5) (1996) 2275-2286.

[109] W.C. Wimley, K. Gawrisch, T.P. Creamer, S.H. White, Proc. Natl. Acad. Sci. U.S.A. 93, (7) (1996) 2985-2990.

[110] E. Wallin, T. Tsukihara, S. Yoshikawa, G. Von-Heijne, A. Elofsson, Protein Sci. 6, (4) (1996) 808-815.

[111] D.A. Marvin, Curr. Opin. Struct. Biol. 8 (1998) 150-158. 